\title{
TNF-a Triggers RIP1/FADD/Caspase-8-Mediated Apoptosis of Astrocytes and RIP3/MLKL-Mediated Necroptosis of Neurons Induced by Angiostrongylus cantonensis Infection
}

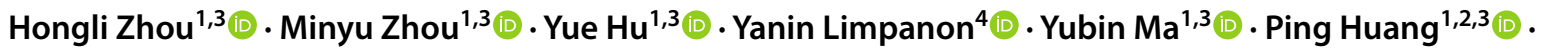 \\ Paron Dekumyoy ${ }^{4}$. $\cdot$ Wanchai Maleewong ${ }^{5}$ (i) $\cdot$ Zhiyue Lv $^{1,2,3}$ (])
}

Received: 5 September 2020 / Accepted: 12 February 2021 / Published online: 8 March 2021

(c) The Author(s) 2021

\begin{abstract}
Angiostrongylus cantonensis (AC) can cause severe eosinophilic meningitis or encephalitis in non-permissive hosts accompanied by apoptosis and necroptosis of brain cells. However, the explicit underlying molecular basis of apoptosis and necroptosis upon AC infection has not yet been elucidated. To determine the specific pathways of apoptosis and necroptosis upon AC infection, gene set enrichment analysis (GSEA) and protein-protein interaction (PPI) analysis for gene expression microarray (accession number: GSE159486) of mouse brain infected by AC revealed that TNF- $\alpha$ likely played a central role in the apoptosis and necroptosis in the context of AC infection, which was further confirmed via an in vivo rescue assay after treating with TNF- $\alpha$ inhibitor. The signalling axes involved in apoptosis and necroptosis were investigated via immunoprecipitation and immunoblotting. Immunofluorescence was used to identify the specific cells that underwent apoptosis or necroptosis. The results showed that TNF- $\alpha$ induced apoptosis of astrocytes through the RIP1/FADD/Caspase- 8 axis and induced necroptosis of neurons by the RIP3/MLKL signalling pathway. In addition, in vitro assay revealed that TNF- $\alpha$ secretion by microglia increased upon LSA stimulation and caused necroptosis of neurons. The present study provided the first evidence that TNF- $\alpha$ was secreted by microglia stimulated by AC infection, which caused cell death via parallel pathways of astrocyte apoptosis (mediated by the RIP1/FADD/caspase- 8 axis) and neuron necroptosis (driven by the RIP3/MLKL complex). Our research comprehensively elucidated the mechanism of cell death after AC infection and provided new insight into targeting TNF- $\alpha$ signalling as a therapeutic strategy for CNS injury.
\end{abstract}

Keywords TNF- $\alpha \cdot$ Angiostrongylus cantonensis $\cdot$ Apoptosis $\cdot$ Necroptosis

Zhiyue Lv

lvzhiyue@mail.sysu.edu.cn

Hongli Zhou

zhouhli7@mail2.sysu.edu.cn

Minyu Zhou

zmylily9787@163.com

Yue $\mathrm{Hu}$

huyue43@mail2.sysu.edu.cn

Yanin Limpanon

yanin.lim@mahidol.ac.th

Yubin Ma

mayb3@mail2.sysu.edu.cn

Ping Huang

huangp33@mail2.sysu.edu.cn

Paron Dekumyoy

paron.dek@mahidol.edu
Wanchai Maleewong

wanch_ma@kku.ac.th

1 Key Laboratory of Tropical Disease Control (Sun Yat-Sen University), Ministry of Education, Guangzhou, China

2 NHC Key Laboratory of Control of Tropical Diseases, Hainan Medical University, Haikou, China

3 Department of Laboratory Medicine, The First Affiliated Hospital, Hainan Medical University, Haikou, China

4 Faculty of Tropical Medicine, Mahidol University, Bangkok, Thailand

5 Faculty of Medicine, Khon Kaen University, Khon Kaen, Thailand 


$\begin{array}{ll}\text { Abbreviations } \\ \text { AC } & \text { Angiostrongylus cantonensis } \\ \text { ACE } & \text { Acetylcysteine } \\ \text { BCA } & \text { Bicinchoninic acid } \\ \text { CNS } & \text { Central nervous system } \\ \text { Ctrl } & \text { Normal control } \\ \text { DEGs } & \text { Differentially expressed genes } \\ \text { dpi } & \text { Days post infection } \\ \text { ECL } & \text { Enhanced chemiluminescence } \\ \text { GSEA } & \text { Gene set enrichment analysis } \\ \text { INF } & \text { Infected by Angiostrongylus cantonensis } \\ \text { LSA } & \text { Larvae soluble antigen } \\ \text { MS } & \text { Mass spectrometry } \\ \text { PFA } & \text { Paraformaldehyde } \\ \text { PPI } & \text { Protein-protein interaction } \\ \text { RIP3 } & \text { Receptor interacting kinase protein3 } \\ \text { RT-qPCR } & \text { Quantitative reverse transcription-polymerase } \\ & \text { chain reaction } \\ \text { TNF- } \alpha & \text { Tumour necrosis factor- } \alpha\end{array}$

\section{Background}

Angiostrongylus cantonensis (hereinafter referred to as AC), also known as rat lungworm, is a metastrongylid pathogen and was first identified in 1935 in Guang Zhou, China (Chen 1935; Hu et al. 2018). After that, additional cases have been reported, especially in underdeveloped countries and territories such as Thailand (Apichat et al. 2016), Oceania and numerous Pacific islands (Lv et al. 2017). AC infection remains the main causative agent of eosinophilic meningitis worldwide (Flerlage et al. 2017). As non-permissive hosts of AC (Ji et al. 2017; Zhou et al. 2019), mice and human beings could be infected by eating raw or uncooked snails containing third-stage larvae, which causes severe eosinophilic meningitis or encephalitis (Mengying et al. 2017). Patients infected by AC suffered from a drastic inflammatory response in the host, neurological impairment and neurodegenerative lesions (Yan et al. 2018; Yoshida et al. 1996), finally leading to memory and cognitive deterioration as a result of the impaired function and irreversible cell death of brain cells (Mengying et al. 2017). To date, the treatment for angiostrongyliasis mainly relies on broad-spectrum antiparasitic drugs such as albendazole and treatment of the symptoms. However, due to a lack of specificity, the pathological outcomes of nasty angiostrongyliasis cannot be markedly improved. Hence, it is urgent to uncover the underlying mechanism of the cell death of brain cells induced by AC infection to provide a new theoretical basis for developing more effective and specific treatment methods.

Tumour necrosis factor- $\alpha$ (TNF- $\alpha)$ is one of the most important pro-inflammatory cytokines (Akash et al. 2018; Al-Gayyar and Elsherbiny 2013), is mainly secreted by macrophage-like cells and functions to regulate the processes of inflammation (Zelova and Hosek 2013), apoptosis (Naimi et al. 2018) and necroptosis (Gunther et al. 2011) by the way of the paracrine system (Lin et al. 2018; Ye et al. 2013). Parasitic infections often lead to the upregulation of $\mathrm{TNF}-\alpha$, which was observed in cases infected with various parasites including A. cantonensis, Leishmania braziliensis (Nieto Gomez et al. 2019; Polari et al. 2019; Schwartz et al. 2018), Plasmodium (Grau and Lou 1995) and Toxoplasma gondii (Park et al. 2019; Pego et al. 2019). As a high level of TNF- $\alpha$ tends to be positively correlated with poor pathological outcomes of patients, an inhibitor of TNF- $\alpha$ has been used to improve the symptoms in some cases (Zhou et al. 2020 ), indicating that TNF- $\alpha$ signalling could be a promising therapeutic target.

Programmed cell death is regulated by specific genes including TNF- $\alpha$ and sequentially activated signalling pathways, among which apoptosis and necroptosis have been well investigated. As reported, TNF- $\alpha$ could induce apoptosis of various cells mainly through RIP1/FADD/ Caspase-8 signalling (Zheng et al. 2016) and could trigger necroptosis of cells via RIP1/RIP3/MLKL signalling (Hu et al. 2020). Many studies have demonstrated that the mechanisms of apoptosis and necroptosis are related to TNF- $\alpha$ in viral infections (Gyurkovska and Ivanovska 2016) and tumours (Balkwill 2006) and promote a treatment target of TNF- $\alpha$ for these diseases (Monaco et al. 2015); however, in parasitic diseases (Barbosa et al. 2018; Carneiro et al. 2018; de Carvalho and Zamboni 2020; Eugenin et al. 2019; Lee et al. 2019; Zamboni and Lima-Junior 2015) much is still unknown. Furthermore, AC is the only neurotropic helminth, and our previous study discovered that the cell death of a mouse brain infected by AC displayed parallel pathways of apoptosis (driven by cleaved caspase-3) and necroptosis [reliant on the host kinase receptor interacting kinase protein3 (RIP3)] (Mengying et al. 2017). However, the explicit molecular basis of apoptosis and necroptosis during AC infection remains to be elucidated.

To illustrate the underlying molecular mechanism of apoptosis and necroptosis in the brain tissue of a non-permissive host infected with $\mathrm{AC}$, gene set enrichment analysis (GSEA) coupled with protein-protein interaction (PPI) network construction analyses was first applied to explore the potential pathways of apoptosis and necroptosis upon AC infection. Mice were treated with a TNF- $\alpha$ inhibitor to investigate the role of TNF- $\alpha$ in apoptosis and necroptosis of mouse brain cells induced by AC. To further depict the specific molecular basis of apoptosis and necroptosis due to AC infection, RT-qPCR, western blot, co-immunoprecipitation and immunofluorescence analyses were applied to explore the potential signalling network of apoptosis and necroptosis in mouse brains, and RT-qPCR, western blot and flow 
cytometry analyses were performed to detect the source of TNF- $\alpha$ after AC infection.

\section{Methods}

\section{Animals}

Twelve female BALB/c mice (6-8 weeks old, $18-20 \mathrm{~g}$ ) were purchased from Charles River Laboratories (Beijing, China) and divided into three groups randomly and equally (4 mice for each group; normal control group: Ctrl; AC infection group: INF; AC infection combined with acetylcysteine treatment group: ACE). Twenty third-stage infectious AC larvae were counted and used to infect the INF and ACE group mice by oral gavage. Additionally, the ACE and INF group mice were, respectively, intraperitoneally injected with acetylcysteine (TNF- $\alpha$ inhibitor, $0.1 \mathrm{mg} / \mathrm{g}$ ) and phosphate-buffered saline (PBS) daily post infection. All the mice were housed in a specific pathogen-free, temperaturecontrolled environment with a $12 \mathrm{~h}$ light/dark cycle.

\section{Parasites and Larvae Soluble Antigen (LSA)}

The infectious third-stage AC larvae were obtained from Biomphalaria glabrata as previously described (Wan et al. 2018) to infect mice, and the young adult AC were harvested from mouse brains 21 days post infection (dpi), washed repeatedly to remove host cells, homogenized on ice to release the soluble antigen, centrifuged at 12,000 rpm and filtered through a $0.22 \mu \mathrm{m}$ sterile filter (Millipore, MA, USA). The protein concentrations of LSA were quantified by a bicinchoninic acid (BCA) kit (Beyotime, Wuhan, China).

\section{GSEA and PPI Network}

To obtain the differentially expressed genes (DEGs) between mouse brain tissues with AC infection and normal control, a gene expression array was performed and data were analysed by Limma package in R language 3.53. Then, GSEA was conducted using the clusterProfiler package (http://www. bioconductor.org/packages/release/bioc/html/clusterProfiler .html) to analyse the critical pathway enriched by AC infection. To identify the hub gene of gene sets in an enriched pathway, PPI analysis was carried out in the STRING database (https://string-db.org/) and visualized by DisGeNET app of Cytoscape software (http://apps.cytoscape.org/apps/ disgenetapp) according to the instructions. All the data of the gene expression microarray involved in the present study have been deposited to GEO database with the accession number of GSE159486.

\section{Cell Lines}

The cell lines used in this study included N9 (mouse microglia cell line) and HT22 (mouse hippocampal neuronal cell line), and N9 was preserved in the Key Laboratory of Tropical Disease Control (Sun Yat-sen University), Ministry of Education and was cultured in Dulbecco's modified Eagle Medium/Nutrient Mixture F-12 medium (Gibco, USA) supplemented with $10 \%$ foetal bovine serum (Gibco, USA) and $100 \mathrm{U} / \mathrm{ml}$ penicillin/streptomycin (Invitrogen, USA). The HT22 cell line was a kind gift from professor Jun Liu in Sun Yat-sen Memorial Hospital and was cultured with Dulbecco's modified Eagle medium (Gibco, USA) plus 10\% foetal bovine serum and $100 \mathrm{U} / \mathrm{ml}$ penicillin/streptomycin. Both cell lines were cultured in a humidified atmosphere containing 5\% $\mathrm{CO}_{2}$ at $37{ }^{\circ} \mathrm{C}$ and were tested for Mycoplasma contamination by RT-PCR regularly. The murine microglial cell line N9 used in this study was derived from mouse brain and was a kind gift from professor Zhongdao Wu (Department of Parasitology, Zhongshan School of Medicine, Sun Yat-sen University, Guangzhou, China).

\section{H\&E Staining}

All mice were sacrificed under anaesthetization (4\% chloral hydrate, intraperitoneal injection, $0.1 \mathrm{ml} / 10 \mathrm{~g}$ ) at 21 days post treatment and the brain tissues were resected, fixed in $4 \%$ paraformaldehyde, paraffin embedded and sectioned into $5 \mu \mathrm{m}$ sections for H\&E staining analysis according to standard procedures as previously described (Ji et al. 2017). Histological configuration of mouse brain tissue was captured, imaged and analysed under an inverted microscope (Lecia, Heidelberg, Germany).

\section{Quantitative Real-Time PCR}

Total RNA was extracted from mouse brain tissues or cell lines using TRIzol Reagent (Invitrogen, Carlsbad, CA) and the purity and concentration of RNA was determined by NanoDrop One (Thermo Fisher scientific, Waltham, USA). The eligible RNA was subjected to a reverse transcriptase reaction with RevertAid First Strand cDNA Kit (Thermo Fisher scientific, Waltham, USA) according to the manufacturer's instructions. Quantitative real-time PCR (RT-qPCR) reactions were amplified for $10 \mathrm{~min}$ at $95^{\circ} \mathrm{C}$ followed by 40 cycles at $95{ }^{\circ} \mathrm{C}$ for $15 \mathrm{~s}$ and $60{ }^{\circ} \mathrm{C}$ for $1 \mathrm{~min}$ with the use of SYBR Green (TaKaRa, Dalian, China) on a LightCycler480 Real-Time PCR System (Roche Diagnostics, Reinach, Switzerland). The relative mRNA expression level of each gene was compared to that of $\beta$-actin (internal control) by the $2^{-\Delta \Delta C t}$ method. The primers used in this study are listed in Table 1. 
Table 1 Primers for qPCR used in this study

\begin{tabular}{lll}
\hline Gene symbol & Forward primer sequence & Reverse primer sequence \\
\hline Caspase-3 & TGGTGATGAAGGGGTCATTTATG & TTCGGCTTTCCAGTCAGACTC \\
Caspase-4 & TGTCATCTCTTTGATATATTCCTGAAG & CAAGGTTGCCCGATCAAT \\
Caspase-6 & AGACAAGCTGGACAACGTGACC & CCAGGAGCCATTCACAGTTTCT \\
Caspase-7 & AAGACGGAGTTGACGCCAAG & CCGCAGAGGCATTTCTCTTC \\
Caspase-8 & TGCTTGGACTACATCCCACAC & TGCAGTCTAGGAAGTTGACCA \\
RIP3 & AAGTGCAGATTGGGAACTACAACTC & AGAATGTTGTGAGCTTCAGGAAGTG \\
RIP1 & GAAGACAGACCTAGACAGCGG & CCAGTAGCTTCACCACTCGAC \\
Fas & TATCAAGGAGGCCCATTTTGC & TGTTTCCACTTCTAAACCATGCT \\
FADD & GCGCCGACACGATCTACTG & TTACCCGCTCACTCAGACTTC \\
TRADD & GGCAGTGCATACCTGTTTTTG & AAATACCCCACTCTCTGACAGT \\
TNF- $\alpha$ & GAACTGGCAGAAGAGGCACT & AGGGTCTGGGCCATAGAACT \\
$\beta$-actin & GCTGTCCCTGTATGCCTCT & GTCTTTACGGATGTCAACG \\
\hline
\end{tabular}

\section{Immunoblotting}

Mouse brain tissues were lysed in RIPA buffer (Thermo Fisher scientific, USA) plus a protease and phosphatase inhibitor cocktail (1:1000, Thermo Fisher scientific, USA) on ice for $5 \mathrm{~min}$, then the lysates were centrifuged at 12,000 rpm for 15 min under $4{ }^{\circ} \mathrm{C}$ and the supernatant was preserved as total protein, the concentration of which was determined by a BCA assay. Next, total protein was denatured at $100{ }^{\circ} \mathrm{C}$ in boiling water for $5 \mathrm{~min}$ and $20 \mu \mathrm{g}$ of denatured protein was subjected to SDS-polyacrylamide gel electrophoresis prior to transfer onto a polyvinylidene fluoride (PVDF, $0.22 \mu \mathrm{m}$ ) membrane (Merck Millipore, MA, USA), which was blocked with 5\% non-fat milk (TBST as solvent) for $2 \mathrm{~h}$ at room temperature before an overnight incubation with the indicated antibodies (list of antibodies is shown in Table 2). After that, HRP-conjugated antibodies (secondary antibodies) were applied and the intensity signals were detected by an enhanced chemiluminescence (ECL) kit (Merck Millipore, MA, USA) according to the manufacturer's instructions. $\beta$-actin or GAPDH was used as an internal control.

\section{Immunofluorescence}

Mouse brain tissues fixed in 4\% paraformaldehyde (PFA) for $24 \mathrm{~h}$ were then dehydrated with $30 \%$ sucrose for $48 \mathrm{~h}$

Table 2 Antibodies

\begin{tabular}{lll}
\hline Antibodies & Source & Identifier \\
\hline Caspase-3 & Cell signaling technology & \#9662 \\
Cleaved caspase-3 (Asp175) (5A1E) rabbit mAb & Cell signaling technology & Cell signaling technology \\
Caspase-8 (D35G2) rabbit mAb & Cell signaling technology \\
Cleaved caspase-8 (Asp387) (D5B2) XP® rabbit mAb (mouse specific) & SANTA CRUZ \\
RIP3 (B-2) mouse mAb & Cell signaling technology \\
Phospho-RIP3 (Thr231/Ser232) (E7S1R) rabbit mAb & Cell signaling technology \\
RIP (D94C12) XP® rabbit mAb & Cell signaling technology \\
Phospho-MLKL (Ser345) (D6E3G) rabbit mAb & Cell signaling technology \\
CYLD (D1A10) rabbit mAb & Cell signaling technology \\
TNF- $\alpha$ (D2D4) XP® rabbit mAb (mouse specific) & Abcam \\
Anti-FADD antibody [EPR5030] & Abways technology \\
GAPDH antibody & Cell signaling technology \\
NeuN (D4G4O) rabbit mAb & Cell signaling technology \\
GFAP (E6N9L) mouse mAb & Cell signaling technology \\
Anti-rabbit IgG, HRP-linked antibody & Cell signaling technology \\
Anti-mouse IgG (H+L), F(ab')2 fragment (Alexa Fluor® 594 conjugate) & Cell signaling technology & \#8592 \\
Anti-rabbit IgG (H+L), F(ab')2 fragment (Alexa Fluor® 488 conjugate) & Abbkine \\
IPKineTM HRP, mouse anti-rabbit IgG LCS & \#3493 \\
\hline
\end{tabular}


and sectioned using a cryostat into $5 \mu \mathrm{m}$ sections. Next, the sections were rewarmed for $30 \mathrm{~min}$, fixed in acetone for 10 min, washed with PBS three times, permeabilized with $0.3 \%$ Triton $\mathrm{X}-100$ for $10 \mathrm{~min}$, incubated with a blocking solution ( $3 \%$ bovine serum albumin in PBS) for $1 \mathrm{~h}$ and then incubated with indicated antibodies at $4{ }^{\circ} \mathrm{C}$ overnight. On the following day, sections were washed three times with PBS (5 min each time), incubated with corresponding secondary antibodies coupled with Alexa Fluor ${ }^{\circledR} 488$ or Alexa Fluor ${ }^{\circledR} 594$ at room temperature for $1 \mathrm{~h}$ under dark conditions, then washed and mounted with 2-(4-amidinophenyl)6-indolecarbamidine dihydrochloride (DAPI) (Beyotime, Wuhan, China). Images of fluorescence were captured with an LSM880 confocal laser-scanning inverted microscope (ZEISS, Jena, German).

\section{Co-immunoprecipitation Assay}

Mouse brain tissues were lysed with IP standard lysis buffer (Thermo Fisher scientific, USA) with protease and a phosphatase inhibitor cocktail (1:1000, Thermo Fisher scientific, USA) for $30 \mathrm{~min}$ at $4{ }^{\circ} \mathrm{C}$ and centrifuged for $15 \mathrm{~min}$ at $12,000 \mathrm{rpm}$. The supernatant was subjected to immunoprecipitation with the indicated antibodies on a rotator for $4 \mathrm{~h}$ at $4{ }^{\circ} \mathrm{C}$. Then, $40 \mu \mathrm{l}$ of agarose-conjugated protein $\mathrm{G}$ beads (Roche, Basel, Switzerland) was added, and the mixture was incubated at $4{ }^{\circ} \mathrm{C}$ overnight. On the next day, the mixture was washed 5 times ( 5 min each time) with pre-cooled PBS containing protease and a phosphatase inhibitor cocktail (Thermo Fisher Scientific, USA). Finally, the proteins on the beads were denatured with $1 \%$ SDS and the supernatant was subjected to immunoblotting or mass spectrometry (MS) determination.

\section{Flow Cytometry}

To analyse the TNF- $\alpha$ expression level of mouse microglia in response to LSA, N9 cells were seeded in the 12-well cell culture plate and stimulated with LSA $(50 \mu \mathrm{g} / \mathrm{ml})$ for 4 and $24 \mathrm{~h}$. In addition, flow cytometry was performed as follows: cells were washed twice in PBS, treated with fixation and permeabilization solution for $20 \mathrm{~min}$ on ice and washed with permeabilized/perm wash buffer (BD Biosciences, CA, USA) according to the manufacturer's protocol. After that, cells were incubated with primary antibodies and a fluorescein-conjugated secondary antibody followed by flow cytometry analysis. To assess the cell death of neurons stimulated with TNF- $\alpha$, HT22 cells were cultured in complete medium with TNF- $\alpha(10 \mathrm{ng} / \mathrm{ml})$ or TNF- $\alpha$ combined with $\mathrm{Z}-\mathrm{VAD}$ for $4 \mathrm{~h}$, stained by Annexin V (Invitrogen, USA) and propidium iodide (PI) and washed three times according to the manufacturer's instructions followed by flow cytometry analysis. The above flow cytometry was conducted on a CytoFLEX flow cytometer (Beckman Coulter, Atlanta, USA) and data were analysed in CytoFLEX.

\section{Statistical Analysis}

In this study, GSEA was conducted via R language (version 3.53, Missouri, USA) and the statistical analysis of the difference was performed with the use of GraphPad Prism 7.00 (San Diego, CA, USA). Data were presented as the means \pm SDs. Student's $t$ tests were used to evaluate significant difference between two independent groups and a $p$ value lower than 0.05 was considered statistically significant.

\section{Results}

\section{GSEA Reveals TNF-a-Related Apoptosis and Necroptosis in the Mouse Brain Upon AC Infection}

In our previous study, the dramatic apoptosis and necroptosis levels were discovered in the parenchyma and hippocampus of the mouse brain upon AC infection (Mengying et al. 2017). To further investigate the underlying mechanisms of apoptosis and necroptosis in the mouse central nervous system (CNS) induced by AC, we first performed gene expression profiles for whole-brain tissues of AC-infected and normal control mice ( $n=2$ mice/each group) and then GSEA was applied via $\mathrm{R}$ language 3.53 to confirm the occurrence of apoptosis and necroptosis. First, we confirmed that all the mice in INF and ACE group successfully developed into angiostrongyliasis after AC infection. Similar to human, mice developed into angiostrongyliasis exhibited impaired neurological function along with imbalanced walking state (Lai et al. 2020). After infection with twenty third-stage infectious AC larvae by oral gavage, mice were monitored the balance capacity by observing the walking balance ability every day. All the mice displayed impaired balance capacity with the symptom of unbalanced walking at eighteen days post infection. Most importantly, immature AC larvae were found on the cerebral surface of all mice in INF and ACE group, providing the most straightforward evidence of successful infection with AC. Next, we performed data analysis for expression profiles and demonstrated that the gene sets of apoptosis and necroptosis in the mouse brains infected by AC were significantly enriched compared with those in the normal control mouse brains (Fig. 1a, b). In total, there were 41 and 48 obviously upregulated core genes markedly enriched in the apoptosis and necroptosis pathway, respectively, as shown in the heatmap (Fig. 1c, d). These core genes were principally associated with the death receptor signalling pathway, and the relative mRNA expression 
a
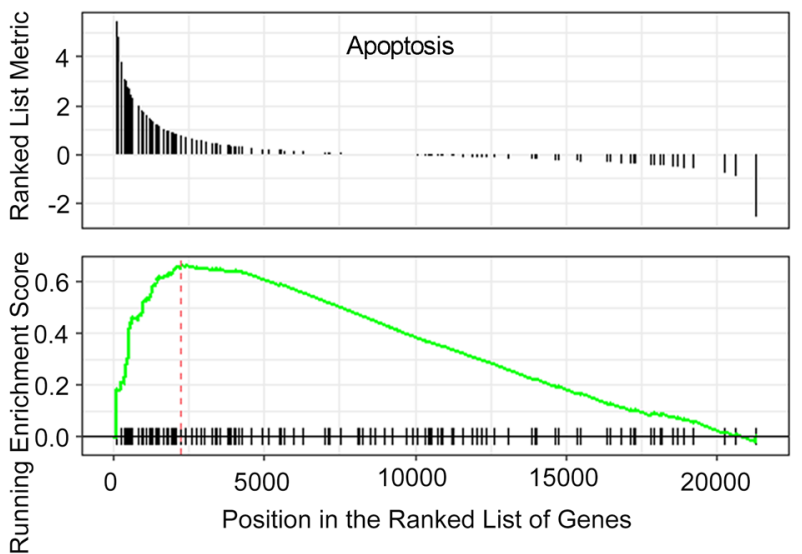

b
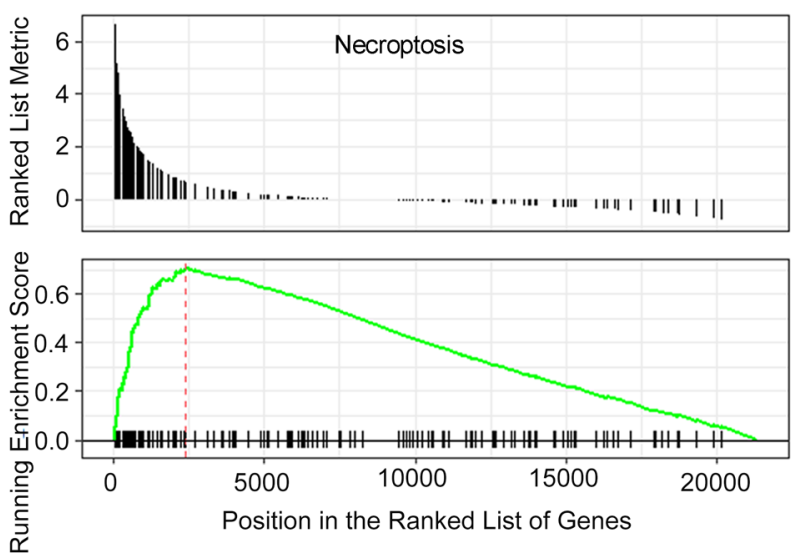

e
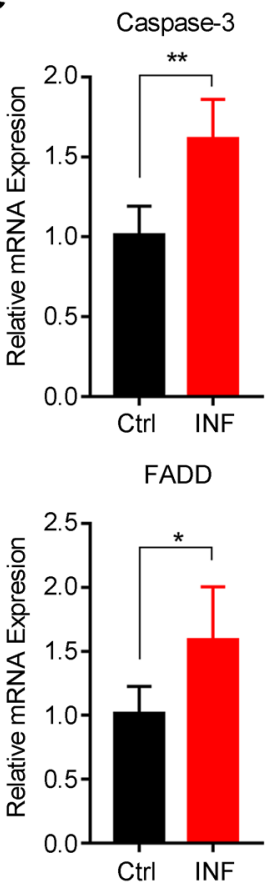

C

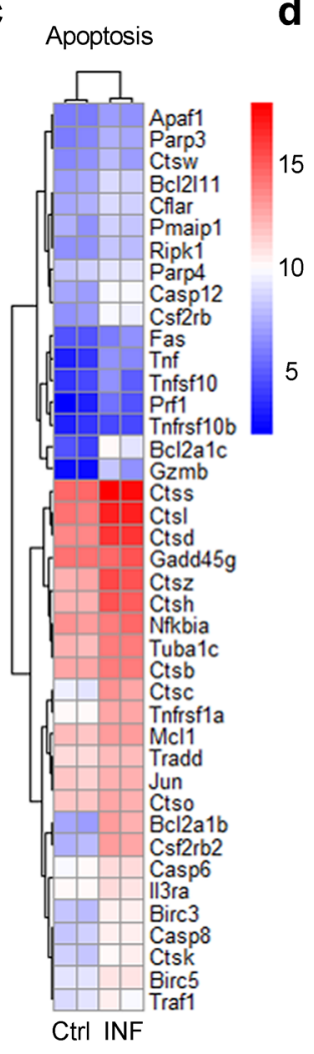

Necroptosis

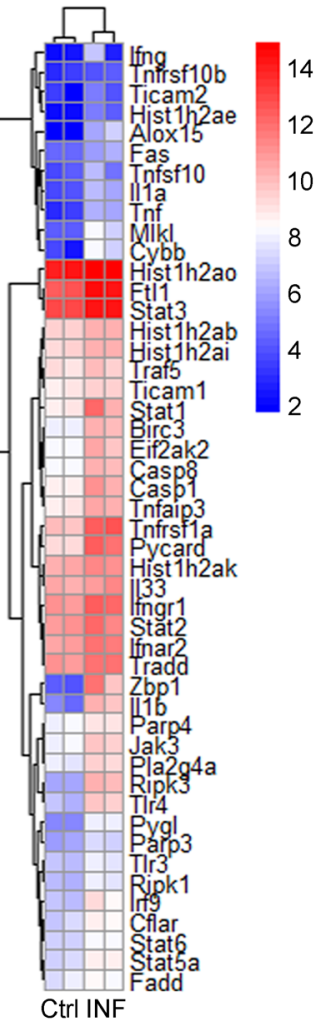

Caspase-7
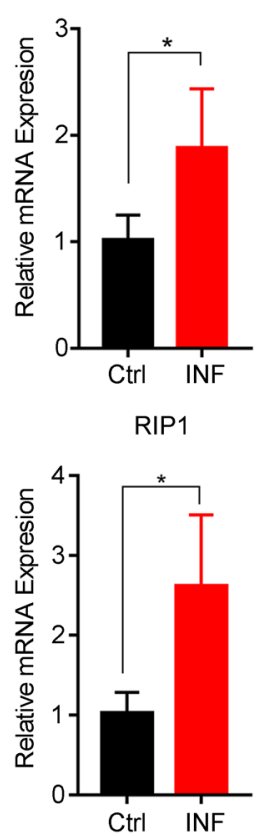

Caspase-8
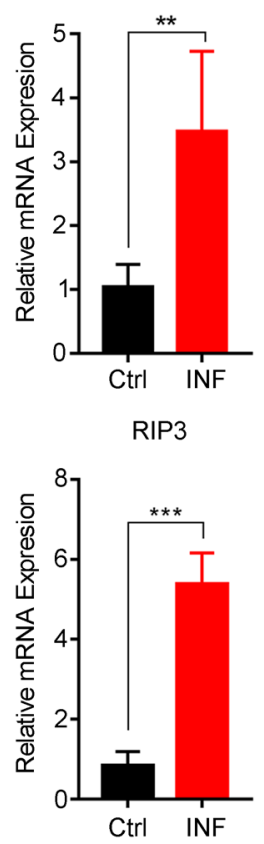
4Fig. 1 GSEA reveals the occurrence of apoptosis and necroptosis in mouse brains upon AC infection. $\mathbf{a}$, $\mathbf{b}$ The brain tissues of normal control and AC-infected mice ( $n=2$ mice/group) were subjected to a gene expression array, and the DEGs between the two groups were further analysed by GSEA, which indicated a significant association of AC infection with apoptosis (a), necroptosis (b) and signalling pathways. c, d Heatmap displayed the relative mRNA expression level of apoptosis and necroptosis pathway-related genes enriched in $\mathbf{a}, \mathbf{b}$. e The DEGs involved in apoptosis and necroptosis were dramatically upregulated in AC-infected mice $(n=4)$ compared with in normal control mice $(n=4)$. * $p<0.05, * * p<0.01$, ***p $p<0.001$ (student's $t$ test). AC Angiostrongylus cantonensis levels of 10 critical genes were checked by RT-qPCR. The results exhibited that the mRNA levels of TRADD, RIP1, caspase-3, caspase-6, and caspase-7 were upregulated twofold compared with those of the normal control; moreover, the mRNA levels of Fas, caspase-8, caspase-4 and RIP3 were fourfold higher than in the normal control (Fig. 1e), which is concordant with the GSEA results. To explore the interactions and hub genes among these core genes of apoptosis and necroptosis, we constructed a PPI network by Cytoscape software and found that caspase- 8 and TNF- $\alpha$ shared most
Fig. 2 The PPI network for the core enriched genes related to the apoptosis and necroptosis pathways. a, b. PPI network analysis for the core enriched genes of GSEA (as shown in Fig. 1c, d) showed that caspase- 8 and TNF- $\alpha$ were the hub genes for the apoptosis and necroptosis pathways in mouse brains infected by AC, respectively. AC Angiostrongylus cantonensis

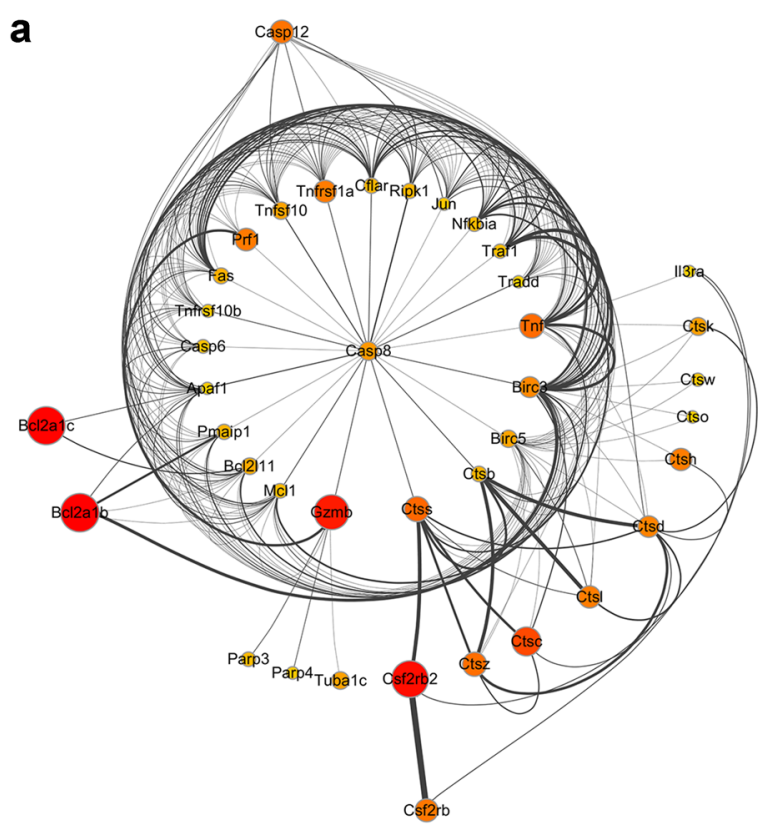

PPI network for core enrichment genes of apoptosis pathay from GSEA analysis
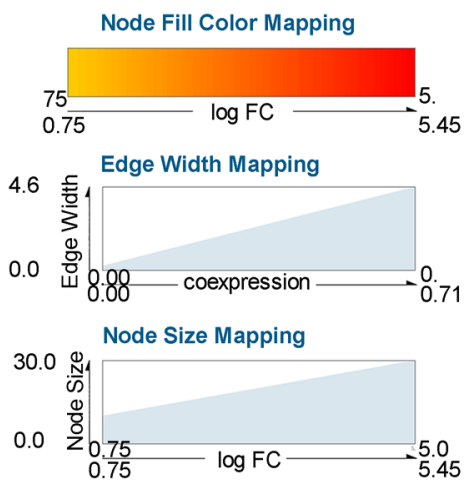

b

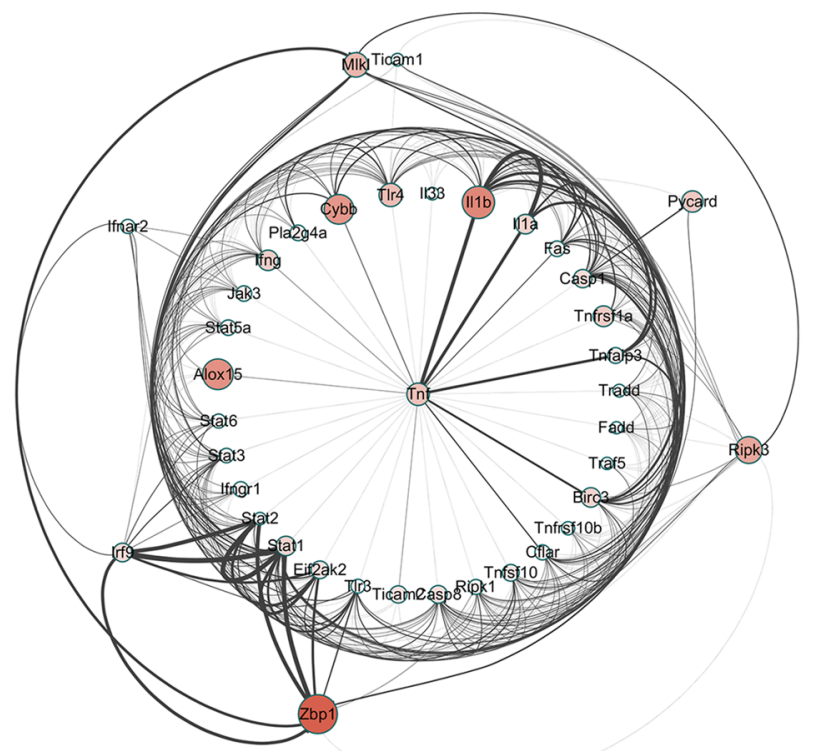

PPI network for core enrichment genes of necroptosis pathay from GSEA analysis
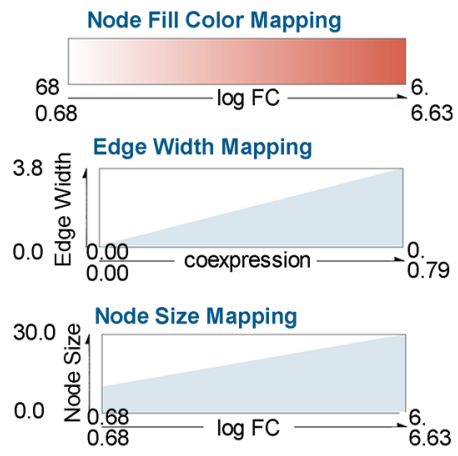
a

C
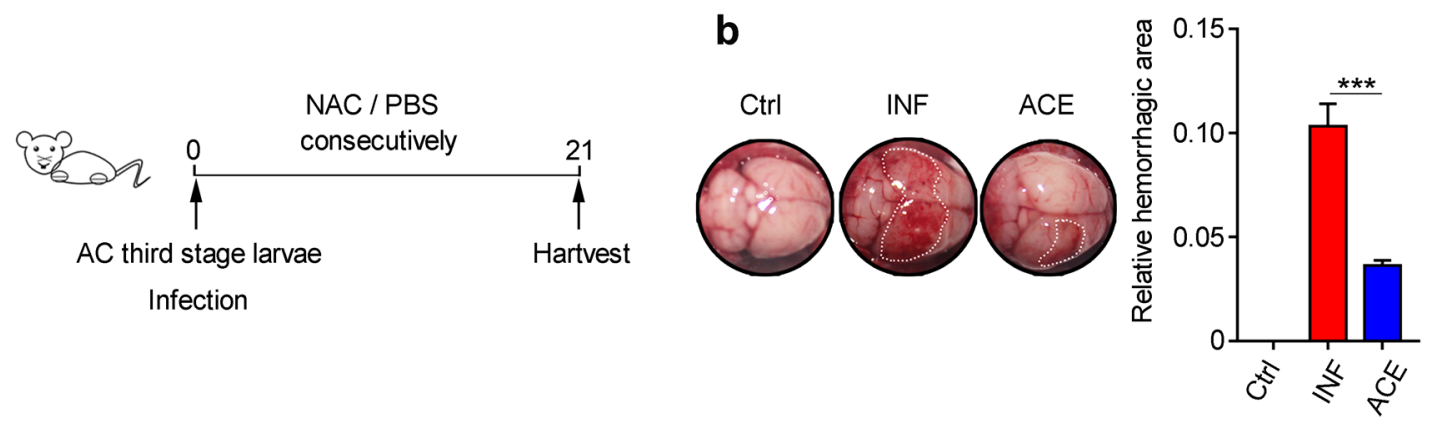
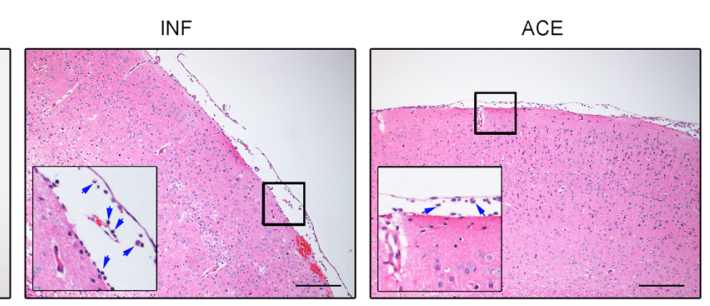

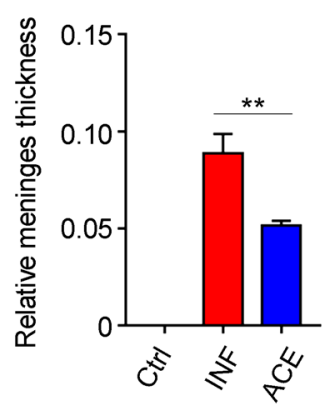

e

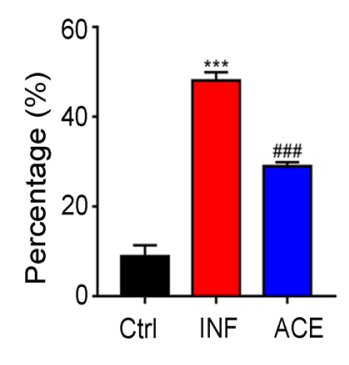

d
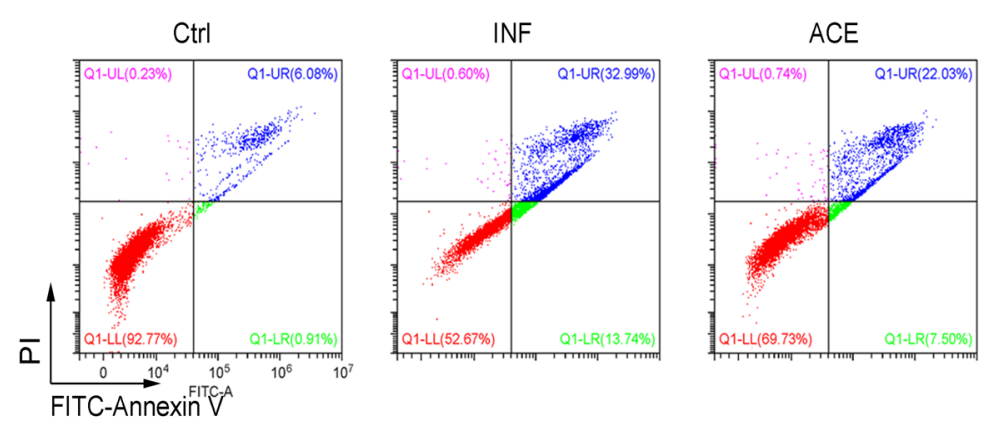

Ctrl INF ACE
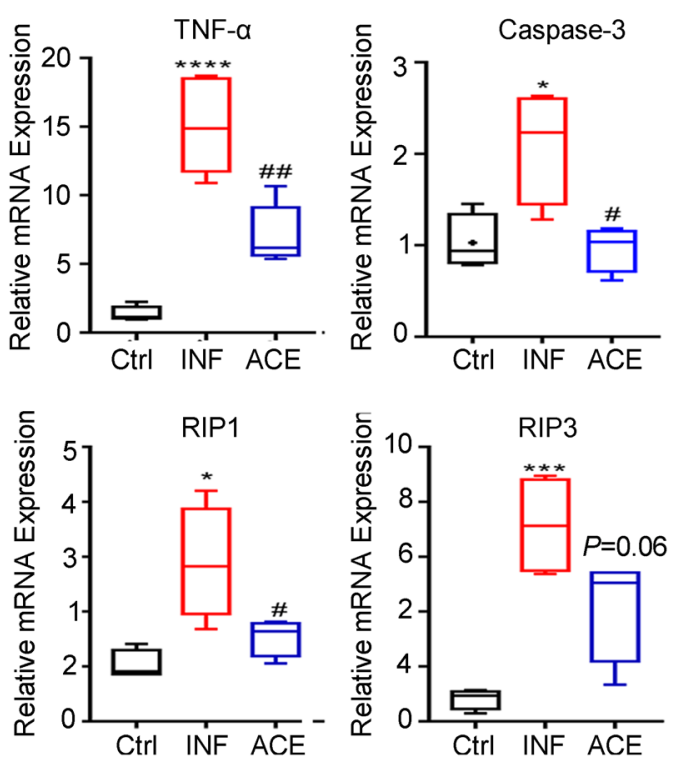

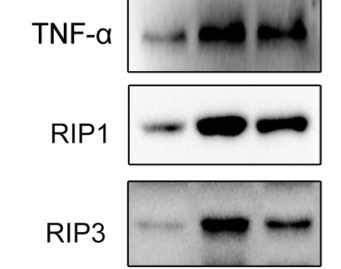

pRIP3

p-MLKL
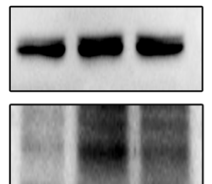

Cleaved
caspase-3
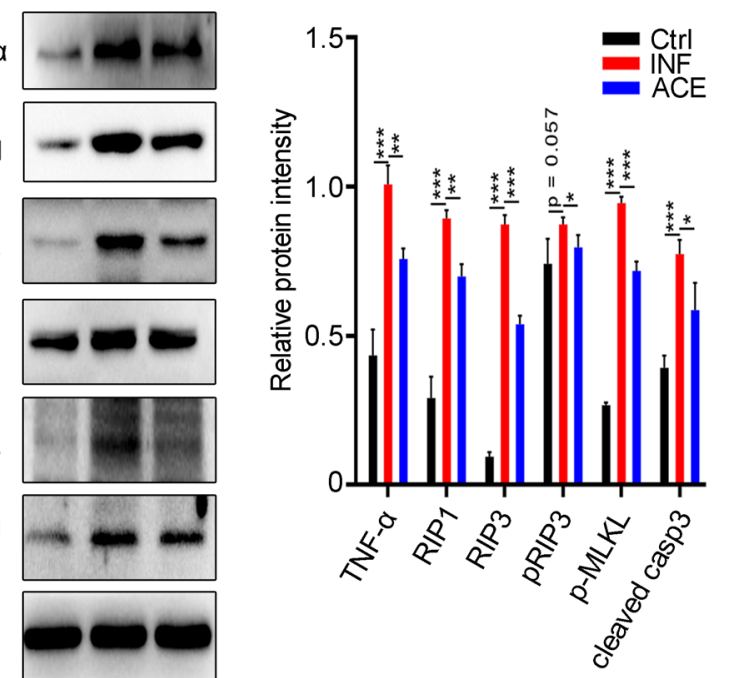
4Fig. 3 Inhibition of TNF- $\alpha$ alleviates the inflammation and cell death of mouse brains with AC infections. a Mice ( $n=4$ per group) were consecutively administered ACE or PBS after AC infection. b Representative images of brain tissues in mice from three groups (left) and quantitative analysis for relative haemorrhagic surface area (right). c Pathological morphology of brain tissues was evaluated by $\mathrm{H} \& \mathrm{E}$ staining (left) and quantitative analysis for relative meninges thickness in H\&E staining (right). d Flow cytometry analysis was used to analyse the cell death of mouse brains. e Percentage of dead cells showed in d. f mRNA levels of TNF- $\alpha$, RIP1, RIP3 and Caspase- 3 were detected after ACE treatment ( $n=4$ mice/group). $\mathbf{g}$ Protein levels of RIP1, RIP3, pRIP3, pMLKL and cleaved caspase-3 in mouse brains of the three groups were examined by western blot (left) and quantitative analysis for protein intensity (right, normalized to GAPDH). ACE acetylcysteine. $* p<0.05$, *** $p<0.001$. $* * * * p<0.0001$ compared to the Ctrl group, \#p<0.05, \#\#p<0.01 compared to the INF group (student's $t$ test)

of the interactions in the apoptosis and necroptosis network, respectively, which demonstrated that caspase- 8 was the hub gene for the apoptosis pathway (Fig. 2a) while TNF- $\alpha$ acted as the hub gene for the necroptosis pathway (Fig. 2b). Furthermore, Caspase- 8 was reported to serve as a downstream gene regulated by TNF- $\alpha$ (Zheng et al. 2016). Collectively, these above data indicated that the apoptosis and necroptosis in mouse brains upon $\mathrm{AC}$ infection was closely related to TNF- $\alpha$.

\section{TNF-a Induces Apoptosis and Necroptosis During AC Infection}

To further investigate whether TNF- $\alpha$ induced apoptosis and necroptosis during AC infection, we conducted a rescue assay in vivo. First, several reported selective inhibitors (Man et al. 2009; Peristeris et al. 1992; Sampaio et al. 1991) of TNF- $\alpha$ (thalidomide, apremilast and acetylcysteine) were tested in our model to evaluate inhibition effects on TNF- $\alpha$ production, among which acetylcysteine (ACE) exhibited the best inhibitory efficacy. Therefore, ACE was chosen as the specific inhibitor of TNF- $\alpha$ in the present study. Next, we divided mice into three groups (normal control: Ctrl; $\mathrm{AC}$ infection: INF; AC infection combined with acetylcysteine treatment: ACE), and the mice of INF and ACE groups were infected with AC. Additionally, the mice of ACE and INF groups were separately treated with acetylcysteine and PBS daily (Fig. 3a). Next, the mice of the three groups were sacrificed at 21 days post infection, and brain tissues were collected for haemorrhagic focus observation and pathological and molecular biological analyses. The results illustrated that $\mathrm{AC}$ infection caused severe haemorrhage in mouse brain tissues compared with normal control while TNF- $\alpha$ inhibitor treatment could reverse the effect (Fig. 3b), implying that TNF- $\alpha$ inhibitor could reduce the inflammatory response induced by AC. In addition, pathological configuration in the mouse brain displayed more thick meninges and more infiltrated inflammatory cells after AC infection, and these characteristics could be obviously attenuated with ACE treatment (Fig. 3c). To explore whether the cell death of brain cells was simultaneously alleviated, we performed flow cytometry and observed that the number of dead cells in the mouse brains of the ACE group were dramatically decreased compared with that in the INF group (Fig. 3d, e). In line with the above result, the mRNA level of a specific marker for apoptosis and necroptosis was increased after AC infection but could be reversed by ACE treatment (Fig. 3f). Moreover, the levels of critical proteins involved in apoptosis (RIP1 and cleaved caspase-3) and necroptosis (RIP3, pRIP3 and pMLKL) in the mouse brains also displayed the expected change upon $\mathrm{AC}$ infection and TNF- $\alpha$ inhibitor treatment, as demonstrated by immunoblotting (Fig. 3g). Hence, TNF- $\alpha$ was confirmed to induce the apoptosis and necroptosis caused by AC.

\section{TNF-a Triggers RIP1/FADD/Caspase-8-Mediated Apoptosis of Astrocytes During AC Infection}

TNF- $\alpha$ acts as a pro-inflammatory cytokine that regulates various signalling pathways such as inflammation (Zelova and Hosek 2013), apoptosis (Naimi et al. 2018) and necroptosis (Gunther et al. 2011). As described above, TNF- $\alpha$ induced apoptosis in mouse brains with AC infections. To demonstrate the specific molecular basis of TNF- $\alpha$ regulating apoptosis in this study, we first detected the transcript level of TNF- $\alpha$ using RT-qPCR, and the result showed that AC-infected mouse brain tissues exhibited a much higher TNF- $\alpha$ mRNA level than normal control mice (Fig. 4a). We further performed immunofluorescence with an antibody against TNF- $\alpha$ in the brain parenchyma and hippocampus of mice infected by $\mathrm{AC}$ and normal control mice to check the protein expression level of TNF- $\alpha$. Not unexpectedly, the fluorescence signal in the AC-infected mouse brain parenchyma and hippocampus was significantly enhanced compared with that in the normal control mouse brain (Fig. $4 \mathrm{~b}$ ), indicating that the TNF- $\alpha$ protein was upregulated upon AC infection. TNF- $\alpha$ could induce pathological and physiological apoptosis by activating various downstream genes, including RIP1, FADD and caspase-8. To identify the specific TNF- $\alpha$ signalling pathway triggering apoptosis in our study, immunoblotting was conducted to detect the expression level of downstream genes of TNF- $\alpha$, and we found that the grey value of TNF- $\alpha$ ( $25 \mathrm{kD}$ and $28 \mathrm{kD}$ ), RIP1 and cleaved caspase- 8 (18 kD and $43 \mathrm{kD})$ substantially accumulated during AC infection, while for total caspase-8, no significant change was observed (Fig. 4c). Furthermore, density analysis for western blot in Fig. $4 \mathrm{c}$ revealed that the protein levels of RIP1, TNF- $\alpha$ and cleaved caspase- 8 (43 $\mathrm{kD}$ and $18 \mathrm{kD}$ ) markedly increased (Fig. 4d-g), of which, RIP1 was enhanced fourfold, indicating that RIP1 could be the main regulator of apoptosis in our study. RIP1 was 

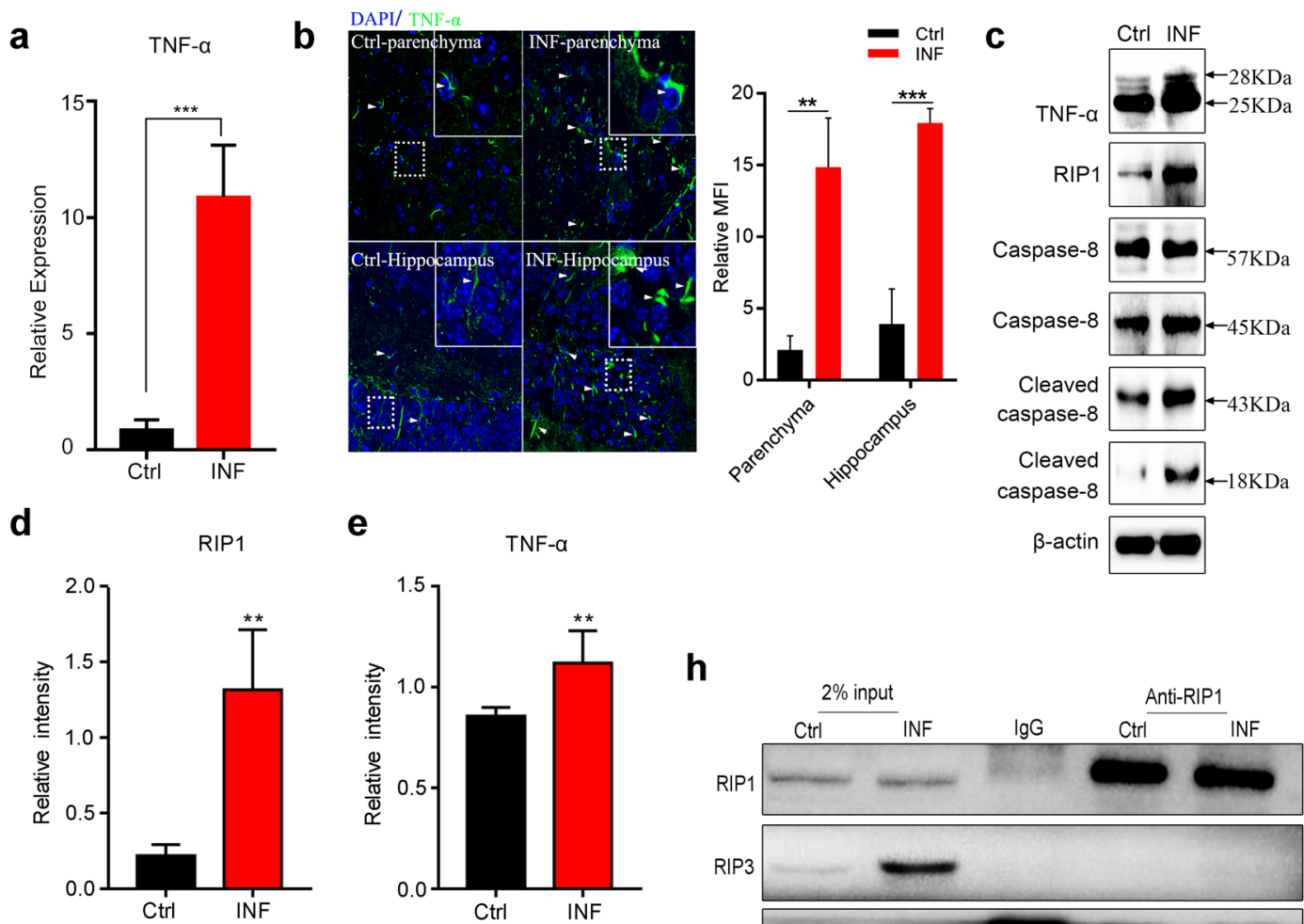

e TNF- $\alpha$

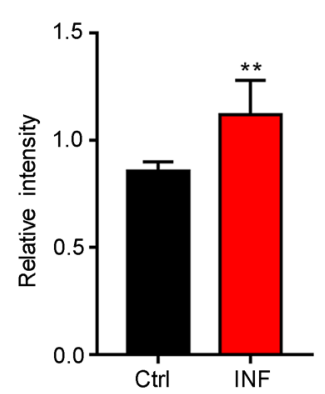

h
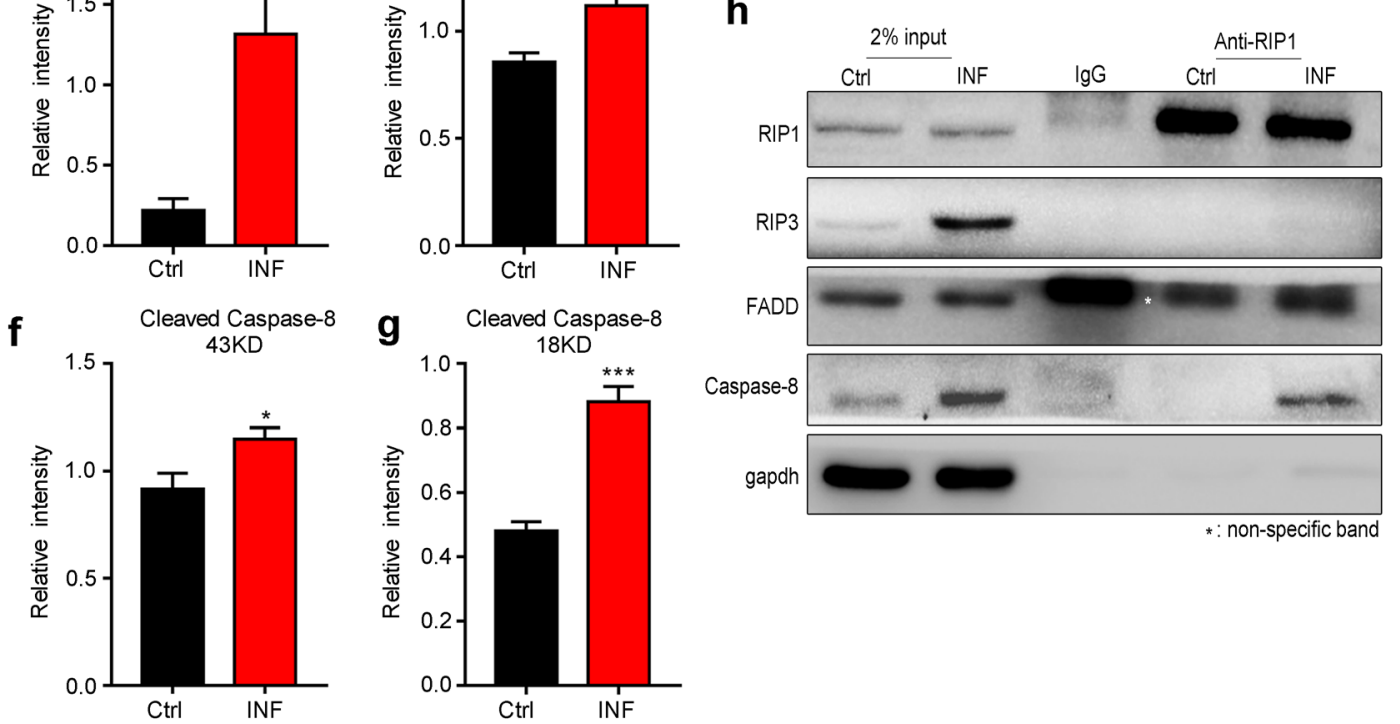

i DAPI/GFAP/Cleaved caspase-3
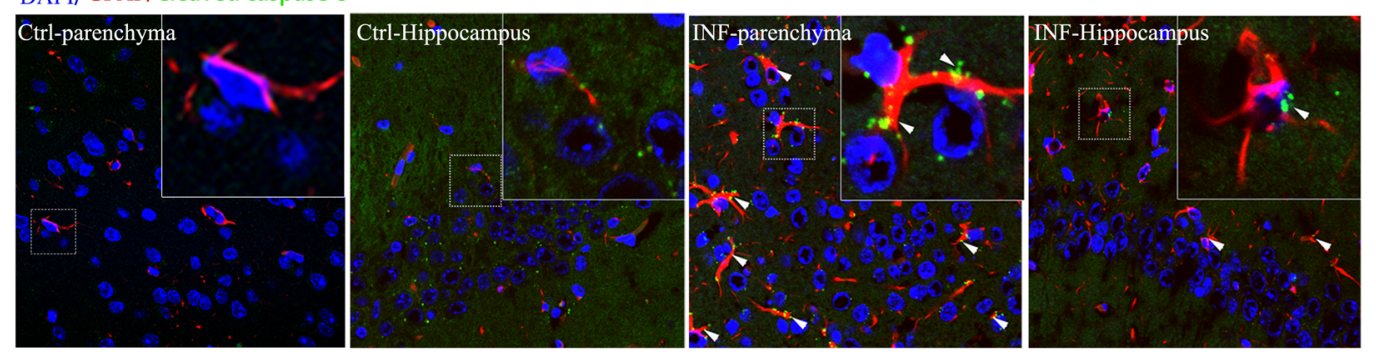

j DAPI/NeuN/Cleaved caspase-3
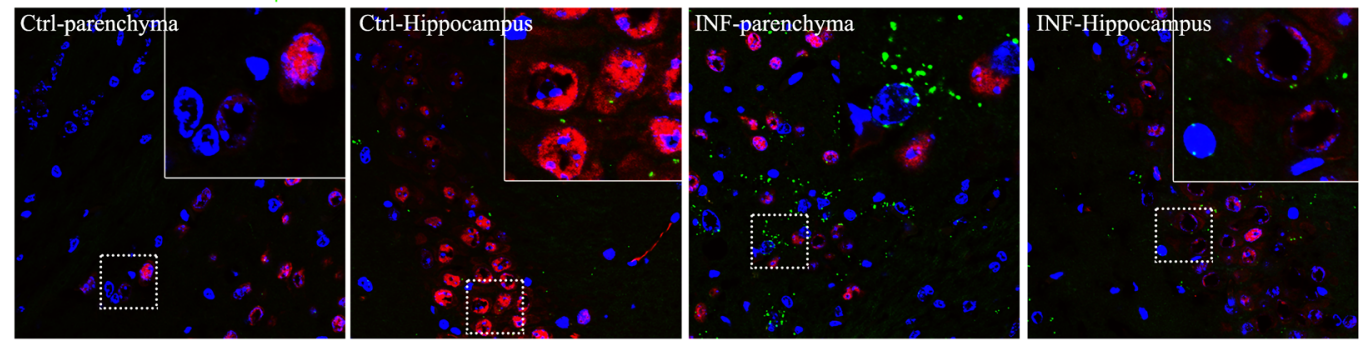
4Fig. 4 TNF- $\alpha$ triggers RIP1/FADD/caspase-8-mediated apoptosis of astrocytes in mouse brains with AC infection. a Relative mRNA level of TNF- $\alpha$ in mouse brains upon AC infection was significantly higher than that in the control group. b TNF- $\alpha$ protein expression level in the mouse brain post infection was remarkably elevated, as revealed by immunofluorescence (left) and relative mean fluorescence intensity (MFI, right). c The lysates of brain tissues from normal control mice or mice post infection were subjected to western blot to determine the protein levels of genes related to the apoptosis signalling pathway (TNF- $\alpha$, RIP1, caspase- 8 and cleaved caspase- 8 ). d-g The protein expression levels in $\mathbf{c}$ were quantified via density analysis. $\mathbf{h}$ Mice were infected by AC for 21 days and then the lysates of mouse brain tissues were immunoprecipitated with an anti-RIP1 antibody or an IgG antibody and the precipitated complexes were separately analysed by immunoblotting with antibodies against RIP1, RIP3, FADD, caspase- 8 and GAPDH. i Cleaved caspase- 3 and GFAP (specific marker of astrocytes) were co-localized, as shown by immunofluorescence. $\mathbf{j}$ Cleaved caspase- 3 and NeuN (specific marker of neurons) showed no co-localization, as shown by immunofluorescence. ${ }^{*} p<0.05,{ }^{*} p<0.01, * * * p<0.001$ (student's $t$ test). AC Angiostrongylus cantonensis, Ctrl normal control, INF infected by Angiostrongylus cantonensis

reported to interact with FADD and caspase-8 under TNF- $\alpha$ stimulation to induce cell apoptosis (Zheng et al. 2016). To confirm whether the TNF- $\alpha /$ RIP1/FADD/caspase-8 signalling pathway was involved in cell apoptosis in our disease model, we performed co-immunoprecipitation using an antiRIP1 antibody and an IgG control antibody for the lysates of the whole-brain tissues from AC-infected mice and normal control mice. With an immunoblotting analysis for the precipitated complex, RIP1 was found to interact directly with FADD and caspase-8 instead of RIP3; moreover, the interaction between RIP1 and caspase- 8 was heightened after AC infection (Fig. 4h), which indicated that TNF- $\alpha$ induced cell apoptosis in our study by activating the RIP1/FADD/ caspase- 8 pathway. As reported, Caspase- 8 functioned in apoptosis progression through Cleaved caspase-3 (executioner of apoptosis), and thus, cleaved caspase- 3 is usually selected as the specific marker for apoptosis (Zheng et al. 2016). Next, to determine the specific cell type during apoptosis in mouse brains with AC infections, we carried out immunofluorescence in the mouse brain parenchyma and hippocampus with an anti-cleaved caspase- 3 antibody and an antibody against a specific cell marker such as GFAP (specific marker for astrocytes) to track a specific cell type in brain tissue. The results show that only GFAP could be colocalized with cleaved caspase- 3 and that the co-localization between GFAP and cleaved caspase- 3 was elevated during AC infection (Fig. 4i), indicating that astrocytes were the main cell type undergoing apoptosis in mouse brains after infection with AC. Moreover, we also examined the apoptosis of other cerebral cells like neurons (Fig. 4j) but failed to observe any significant co-localization of cleaved caspase-3 and NeuN, as shown in Fig. 4j, suggesting that no obvious apoptosis occurred in neurons upon AC infection in mice.
Altogether, TNF- $\alpha$ elicited RIP1/FADD/caspase-8-mediated apoptosis of astrocytes upon $\mathrm{AC}$ infection.

\section{TNF-a Triggers RIP3/MLKL-Mediated Necroptosis of Neurons Upon AC Infection}

In addition to apoptosis, TNF- $\alpha$ additionally induced necroptosis in this study. The signalling pathway involved in necroptosis comprises diverse critical proteins, including CYLD, RIP3, pRIP3 and pMLKL. To verify the existence of necroptosis in our model, we first checked whether the protein levels of these critical proteins related to necroptosis varied in the AC infection group compared with that in the normal control group. With immunoblotting analysis, we found that the grey value of RIP3, pRIP3 and pMLKL was much more intensive in the brain tissues of AC-infected mice than those of normal control (Fig. 5a). Quantitative analysis for the western blot in Fig. 5a revealed that AC infection could significantly elevate the protein levels of RIP3, pRIP3 and pMLKL, but not that of CYLD (Fig. 5b-e). As reported previously, the RIP1/RIP3/MLKL complex was involved in the necroptosis induced by TNF- $\alpha$ (Hu et al. 2020). To explore whether TNF- $\alpha$ induced necroptosis through the same mechanism in our study, a co-immunoprecipitation assay was performed with an anti-RIP3 antibody and an IgG antibody followed by immunoblotting analysis for the precipitated complex. Unexpectedly, the result delineated that RIP3 could bind to MLKL but to neither RIP1 nor FADD (Fig. 5f-g), which was distinct from the reported signalling pathway of necroptosis induced by TNF- $\alpha$ in a reported model. Moreover, to confirm the specific cell type under necroptosis in mouse brains upon $\mathrm{AC}$ infections, we implemented immunofluorescence in the mouse brain parenchyma and hippocampus with antibodies against RIP3 (specific marker for necroptosis) and NeuN (specific marker for neurons) to track a specific cell type in brain tissues. We found that NeuN was overlapped with RIP3 and that the colocalization of NeuN and RIP3 was absent in normal control mouse brain tissues (Fig. 5h), indicating that neurons were undergoing necroptosis in mouse brain tissues. Moreover, we also checked the necroptosis of other non-neuronal cells like astrocytes (Fig. 5i) but failed to find any co-localization between RIP3 and GFAP, indicating that no necroptosis occurred in astrocytes upon AC infection. The above results indicate that TNF- $\alpha$ elicited RIP3/MLKL-mediated necroptosis of neurons in our study.

\section{TNF-a Secreted by Microglia Promotes Necroptosis of Neurons}

As reported previously, $\mathrm{AC}$ is a sort of parasite that stays in mouse brain tissues and dies to form the LSA that stimulates the brain cells to exert a drastic inflammatory response 

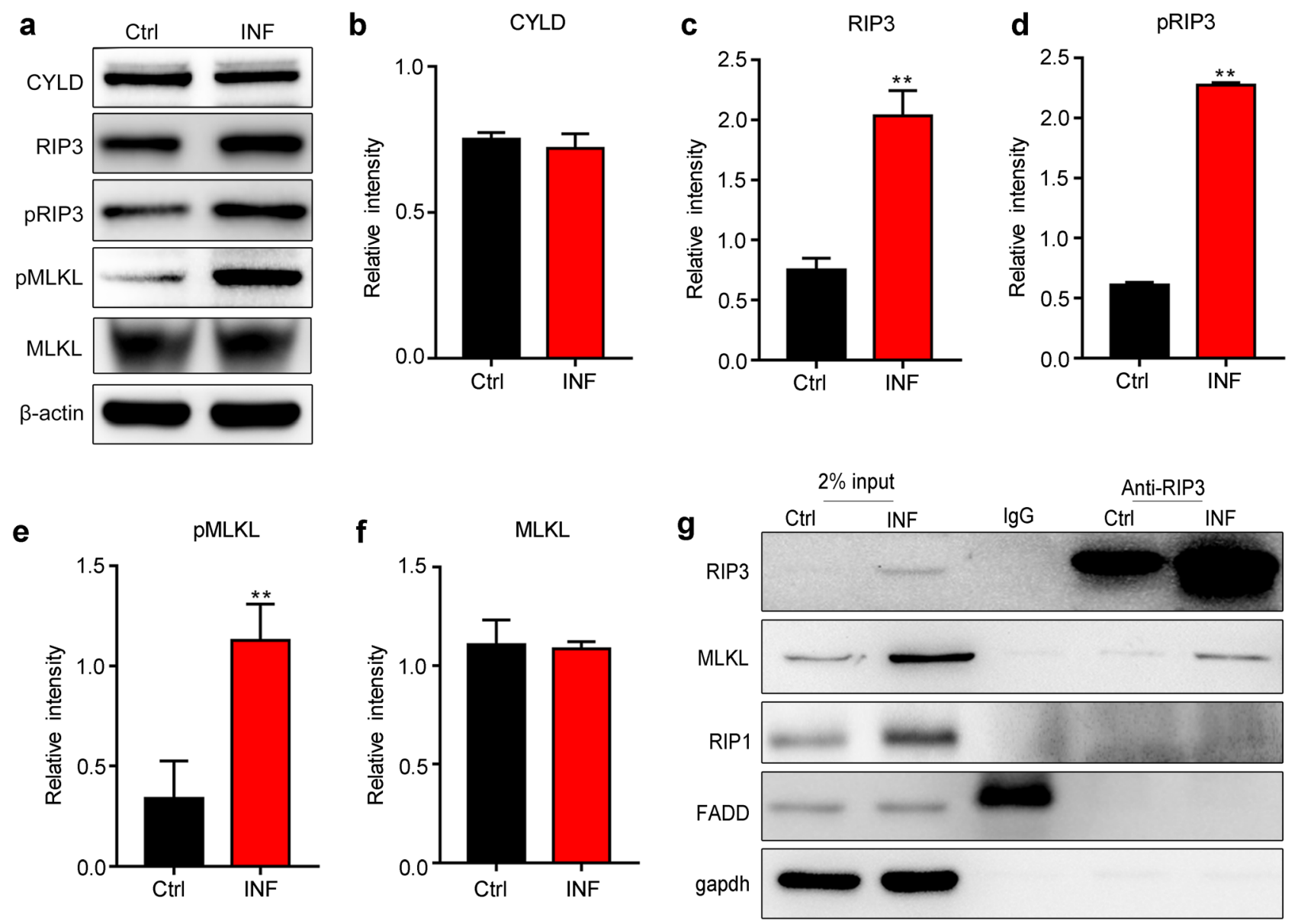

h DAPI/NeuN/RIP3
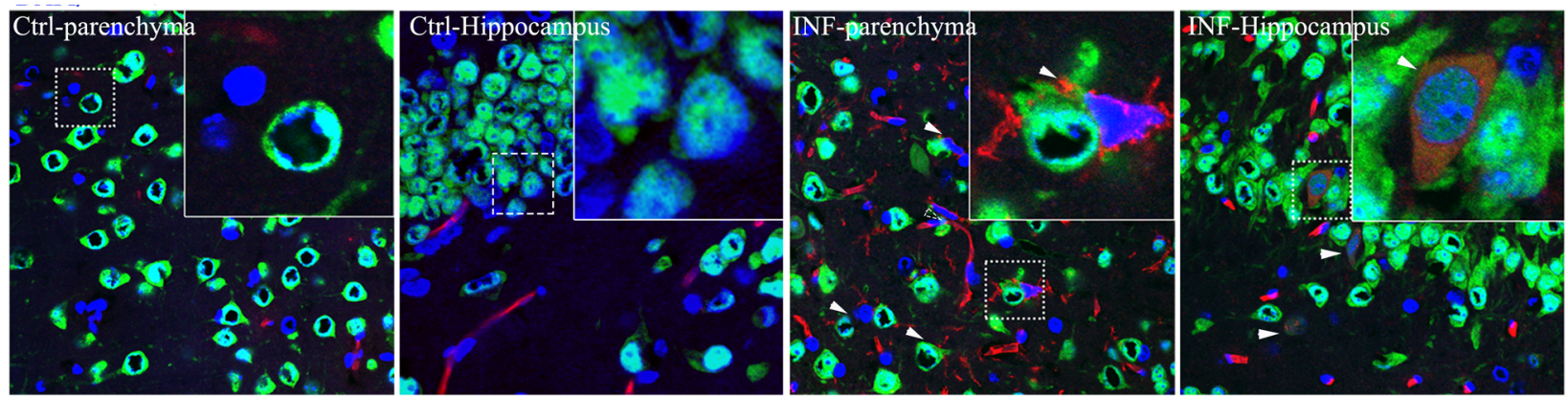

i DAPI/GFAP/RIP3
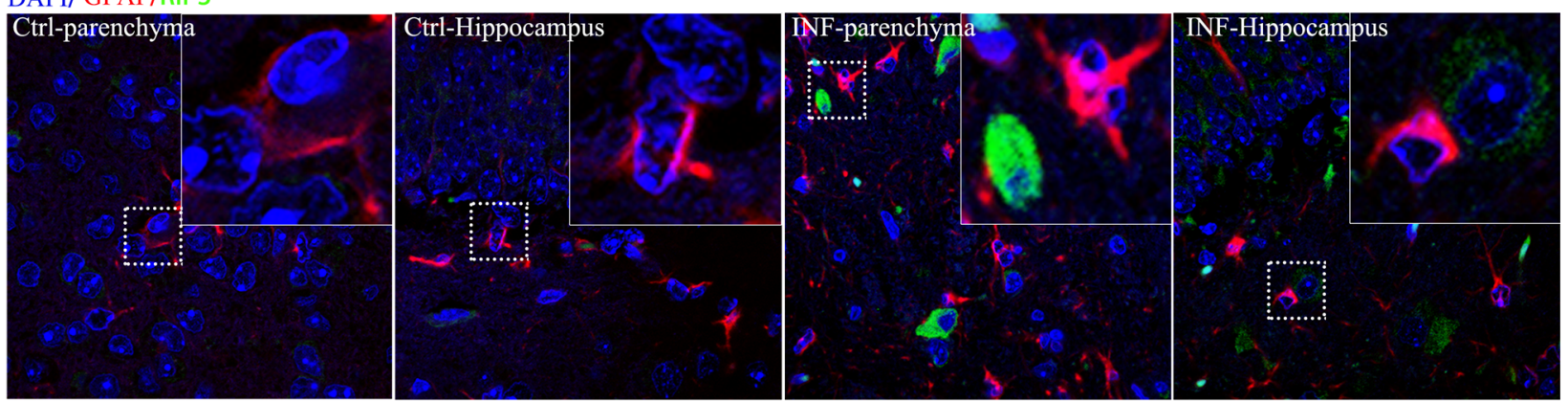
4Fig. 5 TNF- $\alpha$ triggers RIP3/MLKL-mediated necroptosis of neurons in mouse brains infected by AC. a The lysates of brain tissues from normal control mice or mice post infection were subjected to western blot to determine the protein levels of genes related to the necroptosis pathway (RIP3, pRIP3, CYLD and pMLKL). b-f The protein levels in a were quantified via density analysis. $\mathbf{g}$ Mice were infected by AC for 21 days and then the lysates of mouse brain tissues were immunoprecipitated with an anti-RIP3 antibody or an IgG antibody, and the precipitated complexes were separately analysed by immunoblotting with antibodies against RIP3, MLKL, RIP1, FADD and GAPDH, respectively. h RIP3 (specific marker of necroptosis) was co-localized with NeuN (specific marker of neurons), as displayed by immunofluorescence. i RIP3 (specific marker of necroptosis) exhibited no colocalization with GFAP (specific marker of neurons), as showed by immunofluorescence. ${ }^{*} p<0.05, * * p<0.01, * * * p<0.001$ (student's $t$ test). AC Angiostrongylus cantonensis, Ctrl normal control, INF infected by Angiostrongylus cantonensis related to TNF- $\alpha$ (Paouri and Georgopoulos 2019). In this study, TNF- $\alpha$ was confirmed to provoke necroptosis of neurons during $\mathrm{AC}$ infection. However, the cell type in the mouse brain that secreted TNF- $\alpha$ to promote necroptosis of neurons upon AC infection was still unclear. Microglia were the only resident immune cells and the main source of inflammatory cytokines in mouse brain (Hansen et al. 2018). Thus, we surmised that microglia likely secreted TNF- $\alpha$ upon LSA stimulation. To verify our conjecture, we treated mouse microglia (N9 cell line) in vitro with TNF- $\alpha(10 \mathrm{ng} / \mathrm{ml}$, as a positive control) and LSA (50 $\mu \mathrm{g} / \mathrm{ml}$, prepared under aseptic conditions) for 4 and $24 \mathrm{~h}$ to detect the expression level of TNF- $\alpha$. With RT-qPCR, we found that the mRNA level of TNF- $\alpha$ was upregulated by 4.5 -fold and ninefold at 4 and $24 \mathrm{~h}$ after LSA treatment (Fig. 6a). Simultaneously, the protein level
Fig. 6 TNF- $\alpha$ secreted by microglia promotes necroptosis of neurons. a The transcription level of TNF- $\alpha$ in the N9 cell line treated with TNF- $\alpha$ or LSA was much higher than that in control cells. b The protein level of TNF- $\alpha$ in the N9 cell line treated with LSA was significantly higher than that in the control group, as shown by flow cytometry. c Quantitative result of $\mathbf{b}$ showed the protein level of TNF- $\alpha$ in N9. $\mathbf{d}$ The mRNA expression levels of genes related to apoptosis and necroptosis in the mouse hippocampus neuron cell line HT22 treated with LSA for 4 or $24 \mathrm{~h}$ showed no significant change compared to that in control cells, as exhibited by RT-qPCR. e The cell death of the HT22 cell line treated with TNF- $\alpha$ or TNF- $\alpha$ combined with Z-VAD (inhibitor for caspases) was analysed by flow cytometry with triple independent repeats. ${ }^{*} p<0.05$, $* * * p<0.001, * * * * p<0.0001$ (student's $t$ test). LSA larvae soluble antigen a

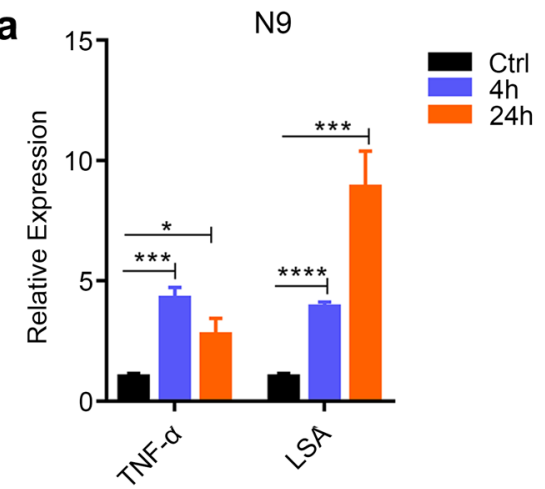

b

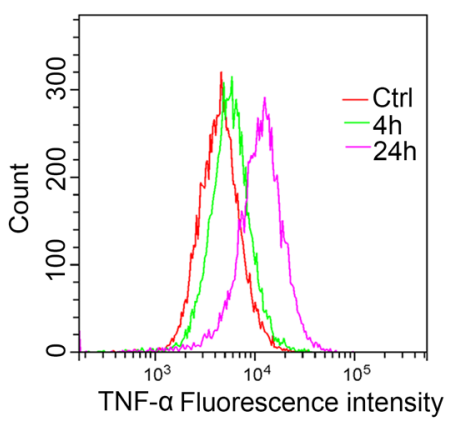

C

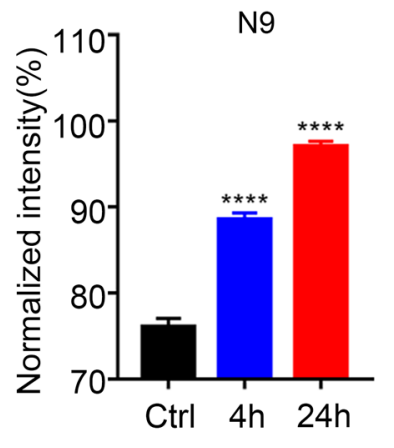

d

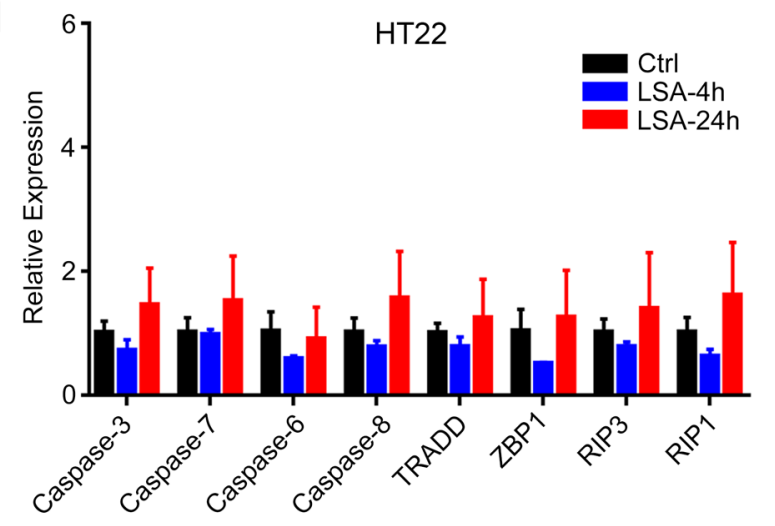

e HT22:TNF- $\alpha \quad$ HT22:TNF- $\alpha+Z-V A D$
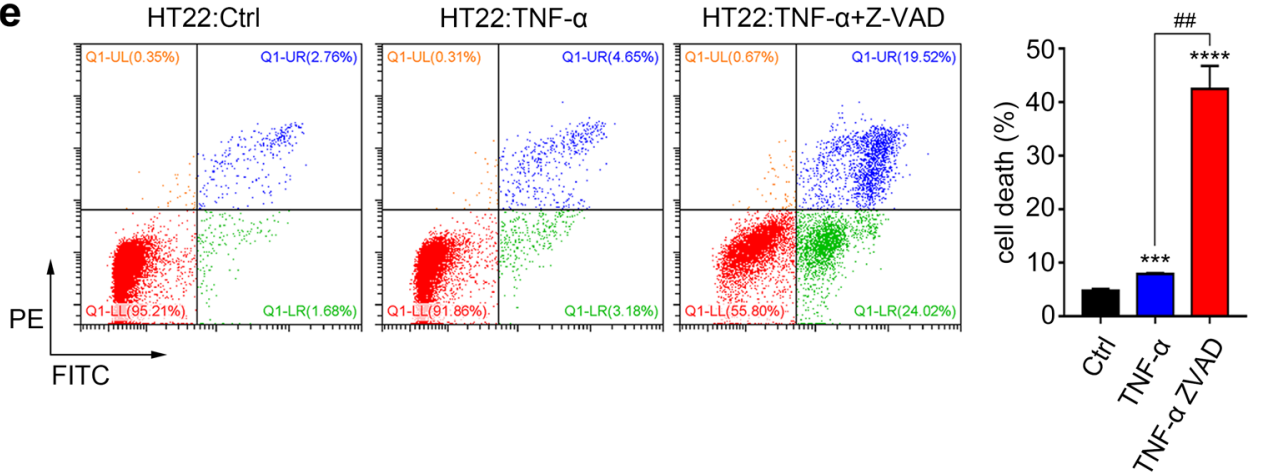
of TNF- $\alpha$ was significantly boosted (Fig. $6 \mathrm{~b}-\mathrm{c}$ ) as exhibited by flow cytometry. Next, we continued to explore whether necroptosis of neurons was directly induced by LSA from dead parasites or TNF- $\alpha$ secreted by activated microglia. First, mouse neurons (HT22 cell line) were treated by LSA ( $50 \mu \mathrm{g} /$ $\mathrm{ml}$ ) for 4 and $24 \mathrm{~h}$ in vitro but failed to provoke a notable upregulation of genes involved in cell death, indicating that LSA could not directly induce the cell death of neurons in our model (Fig. 6d). However, when HT22 cells were stimulated with TNF- $\alpha(10 \mathrm{ng} / \mathrm{ml})$ for $4 \mathrm{~h}$, more dead neurons were observed in the treated group (Fig. 6e) than in the normal control group (7.8\% vs $4.44 \%$ ). In addition, when we treated HT2 2 cells with TNF- $\alpha$ combined with Z-VAD (inhibitor of caspase family), the cell death of neurons was more pronounced (Fig. 6e) than with TNF- $\alpha$ treatment $(43.54 \%$ vs $7.8 \%$ ), revealing that TNF- $\alpha$ could elicit necroptosis of neurons but not apoptosis. Taken together, these results demonstrate that AC infection led to TNF- $\alpha$ secretion by activated microglia to mediate necroptosis of neurons.

\section{Discussion}

Programmed cell death such as apoptosis, necroptosis and autophagy of brain cells plays a pivotal role in neurodegenerative diseases such as Alzheimer's disease, Parkinson's disease, Huntington's disease and irreversible neurological impairment caused by parasitic infection (Yu and $\mathrm{He} 2016$; Yuan et al. 2019). For non-parasitic neurodegenerative disease, the underlying mechanism of cell death in the brain has been well studied. For example, apoptosis of neurons during Alzheimer's disease progression could be induced by PGD2 which stimulated expression of BIK and suppressed expression of ARRB1 (Guo et al. 2017). Neuron autophagy in Parkinson's disease could be regulated by MIR-124 targeting Bim (Wang et al. 2016) while apoptosis of neurons in Huntington's disease was induced by NMDAR-mediated excitotoxicity (Fernandes et al. 2007). As has been reported, an AC infection could cause neurodegenerative symptoms in a mouse model (Ji et al. 2017), and in our previous study, we fully confirmed that AC could induce apoptosis and necroptosis of mouse brain cells (Mengying et al. 2017). However, to date, there are no studies elucidating the specific molecular basis.

In this study, we intended to explore the explicit mechanisms of apoptosis and necroptosis in the mouse brain during AC infection. To this end, we first performed GSEA and PPI network construction analysis on the expression profile of mouse brains with or without AC infection and finally identified TNF- $\alpha$ as the hub gene in the regulatory network of apoptosis and necroptosis; these results imply that TNF- $\alpha$ likely plays a central role in the cell death of mouse brain cells with AC infection. To validate this conjecture, we conducted a rescue assay in vivo, and the result indicated that TNF- $\alpha$ was upregulated after AC infection, which is concordant with the results of a previous study (Chen and Lai 2007), and was downregulated after acetylcysteine treatment. Accordingly, inhibition of TNF- $\alpha$ could reverse the effect of AC infection on inflammation and cell death in the mouse brain, which verified that TNF- $\alpha$ induced apoptosis and necroptosis during $\mathrm{AC}$ infection.

Next, we aimed to further explore the concrete molecular network underlying TNF- $\alpha$-induced apoptosis and necroptosis in our study. For TNF- $\alpha$-induced apoptosis, many studies have demonstrated that the downstream signalling pathway was mainly the RIP1/FADD/Caspase- 8 axis which was characterized by the formation of the RIP1/FADD/Caspase- 8 complex by cleaving Caspase- 8 and then activating Caspase-3 to execute apoptosis (Abhari et al. 2013; Hu et al. 2020; Ikner and Ashkenazi 2011; Long et al. 2019). Therefore, we first investigated whether the same signalling axis was involved in the apoptosis observed in our model. With a co-immunoprecipitation assay for mouse brain tissues, we found that in this study, RIP1 could directly bind to FADD and Caspase- 8 to form a complex, which was in line with the previously reported signalling axis (Abhari et al. 2013; Hu et al. 2020; Ikner and Ashkenazi 2011; Long et al. 2019). Furthermore, the interaction between RIP1 and FADD/Caspase- 8 was notably enhanced after AC infection. In addition, we performed immunofluorescence to identify astrocytes undergoing apoptosis and this was similar to the outcome of astrocytes in rats infected with AC (Zhou et al. 2019). For TNF- $\alpha$-induced necroptosis, previous studies indicated that the downstream signalling axis was the RIP1/RIP3/MLKL pathway characterized by the formation of the RIP1/RIP3/ MLKL complex (Wang et al. 2019; Zhang et al. 2018). To elucidate the specific signalling pathway regulated by TNF- $\alpha$ in our study, we also conducted co-immunoprecipitation using mouse brain tissues and unexpectedly discovered that RIP3 merely bound to MLKL, which was distinct from the canonical signalling axis described above. Similarly, the RIP3/MLKL complex was also discovered to be involved in necroptosis due to murine cytomegalovirus and influenza A virus infections (Thapa et al. 2016; Upton et al. 2019); in the absence of RIPK1, RIP3/MLKL triggers necroptosis of cells induced by IFNs (Ingram et al. 2019; Upton et al. 2019). Thus, we speculate that there should be some similar mechanism leading to necroptosis between parasite and virus infections. Finally, we confirmed necroptosis of neurons induced by AC via immunofluorescence. Although through the in vivo experiments above, we generally could draw the conclusion that TNF- $\alpha$ drove apoptosis of astrocytes and necroptosis of neurons after AC infection, inclusion of another group of mice treated with TNF- $\alpha$ inhibitor will better confirm this conclusion. 
As a pro-inflammatory cytokine, TNF- $\alpha$ is mainly secreted by cells involved in the inflammatory response, including macrophages, natural killer (NK) cells, and lymphocytes (Wang et al. 2012, 2018). As is known, in brain tissue, microglia act as the sole resident immune cell, thus, we surmised that the high level of TNF- $\alpha$ was produced by microglia upon AC infection. To verify this assumption, we performed an in vitro assay to stimulate N9 (mouse microglia cell line) with LSA, and the result confirmed that AC infection could induce microglia to secret TNF$\alpha$. Recently, microglial TNF- $\alpha$ was reported to be elevated under pathological conditions, which was in concordance with our results (Borrajo et al. 2014; Lewitus et al. 2016). However, the increased microglial TNF- $\alpha$ had diverse functions, including suppressing neuronal plasticity (Lewitus et al. 2016) and mediating dopaminergic degeneration. Dissimilarly, in this study, upregulation of microglial TNF- $\alpha$ could induce RIP3/MLKL-mediated necroptosis of neurons, which expanded our knowledge of the role of microglia.

\section{Conclusion}

In summary, the present study first illustrated that the TNF- $\alpha$ secreted by microglia during AC infection induced RIP1/ FADD/caspase-8-mediated apoptosis of astrocytes and RIP3/MLKL-mediated necroptosis of neurons. Our research comprehensively elucidated the mechanism of cell death after AC infection and provided new insight into targeting TNF- $\alpha$ signalling for therapeutic strategies during CNS injury.

Author Contributions This project was designed and conceived by LZY. LZY and ZHL wrote the article. Experiments were performed by ZHL, ZMY and HY. LY, HP and MYZ analysed data and prepared figures and tables. DP and MW participated in study design and coordination. All authors read and approved the final paper.

Funding This work was supported by grants from the Major Science and Technology Program of Hainan Province (Grant No. ZDKJ202003), the National Natural Science Foundation of China (Grant Nos. 82072303 and 81572023), the Key Research and Development Program of Hainan Province (Grant No. ZDYF2020120), the Guangdong Natural Science Foundation (Grant No. 2019A1515011541), the Science and Technology Planning Project of Guangdong Province (Grant No. 2019B030316025), the National Parasitic Resources Center of China (Grant No. NPRC-2019-194-30), the Open Foundation of Key Laboratory of Tropical Translational Medicine of Ministry of Education, Hainan Medical University (Grant No. 2020TTM007) and the 111 Project (Grant No. B12003).

Data Availability The data supporting the conclusions of this article are included within the article and its additional files.

\section{Compliance with Ethical Standards}

Conflict of interest The authors declare that they have no conflict of interest.

Ethical Approval All animal experiments were approved by the Medical Research Ethics Committee of Sun Yat-sen University and conformed to the Chinese National Institute of Health Guide for the Care and Use of Laboratory Animals (NO. 2017-166).

Open Access This article is licensed under a Creative Commons Attribution 4.0 International License, which permits use, sharing, adaptation, distribution and reproduction in any medium or format, as long as you give appropriate credit to the original author(s) and the source, provide a link to the Creative Commons licence, and indicate if changes were made. The images or other third party material in this article are included in the article's Creative Commons licence, unless indicated otherwise in a credit line to the material. If material is not included in the article's Creative Commons licence and your intended use is not permitted by statutory regulation or exceeds the permitted use, you will need to obtain permission directly from the copyright holder. To view a copy of this licence, visit http://creativecommons.org/licenses/by/4.0/.

\section{References}

Abhari BA, Cristofanon S, Kappler R, von Schweinitz D, Humphreys R, Fulda S (2013) RIP1 is required for IAP inhibitor-mediated sensitization for TRAIL-induced apoptosis via a RIP1/FADD/ caspase-8 cell death complex. Oncogene 32:3263-3273. https:// doi.org/10.1038/onc.2012.337

Akash MSH, Rehman K, Liaqat A (2018) Tumor necrosis factor-alpha: role in development of insulin resistance and pathogenesis of type 2 diabetes mellitus. J Cell Biochem 119:105-110. https://doi. org $/ 10.1002 / j \mathrm{cb} .26174$

Al-Gayyar MM, Elsherbiny NM (2013) Contribution of TNF-alpha to the development of retinal neurodegenerative disorders. Eur Cytokine Netw 24:27-36. https://doi.org/10.1684/ecn.2013.0334

Apichat V et al (2016) Phylogeny of angiostrongylus cantonensis in Thailand based on cytochrome $\mathrm{C}$ oxidase subunit I gene sequence Southeast Asian. J Trop Med Public Health 47:377-386

Balkwill F (2006) TNF-alpha in promotion and progression of cancer. Cancer Metastasis Rev 25:409-416. https://doi.org/10.1007/s1055 5-006-9005-3

Barbosa LA et al (2018) RIPK1-RIPK3-MLKL-associated necroptosis drives leishmania infantum killing in neutrophils. Front Immunol 9:1818. https://doi.org/10.3389/fimmu.2018.01818

Borrajo A, Rodriguez-Perez AI, Diaz-Ruiz C, Guerra MJ, LabandeiraGarcia JL (2014) Microglial TNF-alpha mediates enhancement of dopaminergic degeneration by brain angiotensin. Glia 62:145157. https://doi.org/10.1002/glia.22595

Carneiro MBH et al (2018) NOX2-derived reactive oxygen species control inflammation during leishmania amazonensis infection by mediating infection-induced neutrophil apoptosis. J Immunol 200:196-208. https://doi.org/10.4049/jimmunol.1700899

Chen HT (1935) A new pulmonary nematode of rats, Pulmonema cantonensis ng.nsp from Canton. Ann Parasitol 13:312-317

Chen KM, Lai SC (2007) Biochemical and pathological evaluation of albendazole/thalidomide co-therapy against eosinophilic meningitis or meningoencephalitis induced by Angiostrongylus cantonensis. J Antimicrob Chemother 59:264-276. https://doi.org/10.1093/ jac/dk1492 
de Carvalho RVH, Zamboni DS (2020) Inflammasome activation in response to intracellular protozoan parasites. Trends Parasitol 36:459-472. https://doi.org/10.1016/j.pt.2020.02.006

Eugenin EA, Martiney JA, Berman JW (2019) The malaria toxin hemozoin induces apoptosis in human neurons and astrocytes: Potential role in the pathogenesis of cerebral malaria. Brain Res 1720:146317. https://doi.org/10.1016/j.brainres.2019.146317

Fernandes HB, Baimbridge KG, Church J, Hayden MR, Raymond LA (2007) Mitochondrial sensitivity and altered calcium handling underlie enhanced NMDA-induced apoptosis in YAC128 model of Huntington's disease. J Neurosci 27:13614-13623. https://doi. org/10.1523/JNEUROSCI.3455-07.2007

Flerlage T, Qvarnstrom Y, Noh J, Devincenzo JP, Madni A, Bagga B, Hysmith ND (2017) Angiostrongylus cantonensis eosinophilic meningitis in an infant, Tennessee, USA. Emerg Infect Dis 23:1756-1758. https://doi.org/10.3201/eid2310.170978

Grau GE, Lou JN (1995) Experimental cerebral malaria: possible new mechanisms in the TNF-induced microvascular pathology. Soz Praventivmed 40:50-57. https://doi.org/10.1007/BF01615662

Gunther C et al (2011) Caspase-8 regulates TNF-alpha-induced epithelial necroptosis and terminal ileitis. Nature 477:335-339. https:// doi.org/10.1038/nature 10400

Guo JW, Guan PP, Ding WY, Wang SL, Huang XS, Wang ZY, Wang P (2017) Erythrocyte membrane-encapsulated celecoxib improves the cognitive decline of Alzheimer's disease by concurrently inducing neurogenesis and reducing apoptosis in APP/PS1 transgenic mice. Biomaterials 145:106-127. https://doi.org/10.1016/j. biomaterials.2017.07.023

Gyurkovska V, Ivanovska N (2016) Distinct roles of TNF-related apoptosis-inducing ligand (TRAIL) in viral and bacterial infections: from pathogenesis to pathogen clearance. Inflamm Res 65:427437. https://doi.org/10.1007/s00011-016-0934-1

Hansen DV, Hanson JE, Sheng M (2018) Microglia in Alzheimer's disease. J Cell Biol 217:459-472. https://doi.org/10.1083/jcb.20170 9069

Hu QA et al (2018) Small-scale spatial analysis of intermediate and definitive hosts of Angiostrongylus cantonensis. Infect Dis Poverty 7:100. https://doi.org/10.1186/s40249-018-0482-8

Hu S, Chang X, Zhu H, Wang D, Chen G (2020) PI3K mediates tumor necrosis factor induced-necroptosis through initiating RIP1-RIP3MLKL signaling pathway activation. Cytokine 129:155046. https ://doi.org/10.1016/j.cyto.2020.155046

Ikner A, Ashkenazi A (2011) TWEAK induces apoptosis through a death-signaling complex comprising receptor-interacting protein 1 (RIP1), Fas-associated death domain (FADD), and caspase-8. J Biol Chem 286:21546-21554. https://doi.org/10.1074/jbc. M110.203745

Ingram JP et al (2019) ZBP1/DAI drives RIPK3-mediated cell death induced by IFNs in the absence of RIPK1. J Immunol 203:13481355. https://doi.org/10.4049/jimmunol.1900216

Ji L et al (2017) Study on the tolerance and adaptation of rats to Angiostrongylus cantonensis infection. Parasitol Res 116:1937-1945. https://doi.org/10.1007/s00436-017-5472-4

Lai SC, Lu CY, Shyu LY, Chen KM (2020) Angiostrongylus cantonensis infection induces MMP-9 and causes tight junction protein disruption associated with Purkinje cell degeneration. Parasitol Res 119:3433-3441. https://doi.org/10.1007/s00436-020-06840-y

Lee SH, Chu KB, Quan FS (2019) Parasite infiltration and apoptosis in spleen upon toxoplasma gondii infection. Korean J Parasitol 57:537-541. https://doi.org/10.3347/kjp.2019.57.5.537

Lewitus GM, Konefal SC, Greenhalgh AD, Pribiag H, Augereau K, Stellwagen D (2016) Microglial TNF-alpha suppresses cocaineinduced plasticity and behavioral sensitization. Neuron 90:483491. https://doi.org/10.1016/j.neuron.2016.03.030

Lin SH, Chuang HY, Ho JC, Lee CH, Hsiao CC (2018) Treatment with TNF-alpha inhibitor rectifies M1 macrophage polarization from blood CD14+ monocytes in patients with psoriasis independent of STAT1 and IRF-1 activation. J Dermatol Sci 91:276-284. https ://doi.org/10.1016/j.jdermsci.2018.05.009

Long J et al (2019) JAG2/Notch2 inhibits intervertebral disc degeneration by modulating cell proliferation, apoptosis, and extracellular matrix. Arthritis Res Ther 21:213. https://doi.org/10.1186/s1307 5-019-1990-Z

Lv S, Zhou XN, Andrews JR (2017) Eosinophilic meningitis caused by Angiostrongylus cantonensis. ACS Chem Neurosci 8:1815-1816. https://doi.org/10.1021/acschemneuro.7b00233

Man HW et al (2009) Discovery of (S)-N-[2-[1-(3-ethoxy4-methoxyphenyl)-2-methanesulfonylethyl]-1,3-dioxo-2,3-dihy dro-1H-isoindol-4-yl] acetamide (apremilast), a potent and orally active phosphodiesterase 4 and tumor necrosis factor-alpha inhibitor. J Med Chem 52:1522-1524. https://doi.org/10.1021/jm900 $210 \mathrm{~d}$

Mengying $\mathrm{Z}$ et al (2017) Apoptosis and necroptosis of mouse hippocampal and parenchymal astrocytes, microglia and neurons caused by Angiostrongylus cantonensis infection. Parasit Vectors 10:611. https://doi.org/10.1186/s13071-017-2565-y

Monaco C, Nanchahal J, Taylor P, Feldmann M (2015) Anti-TNF therapy: past, present and future. Int Immunol 27:55-62. https:// doi.org/10.1093/intimm/dxu102

Naimi A, Movassaghpour AA, Hagh MF, Talebi M, Entezari A, JadidiNiaragh F, Solali S (2018) TNF-related apoptosis-inducing ligand (TRAIL) as the potential therapeutic target in hematological malignancies. Biomed Pharmacother 98:566-576. https://doi. org/10.1016/j.biopha.2017.12.082

Nieto Gomez P, Casas Hidalgo I, Casas Hidalgo MP, Alvarez Sanchez R, Rodriguez Delgado A, Cabeza-Barrera J (2019) Cutaneous leishmaniasis associated with TNF-alpha blockers: a case report. Eur J Hosp Pharm 26:233-234. https://doi.org/10.1136/ejhpharm2018-001521

Paouri E, Georgopoulos S (2019) Systemic and CNS inflammation crosstalk: implications for Alzheimer's disease. Curr Alzheimer Res 16:559-574. https://doi.org/10.2174/15672050166661903211 54618

Park EA, Han IH, Kim JH, Park SJ, Ryu JS, Ahn MH (2019) Production of inflammatory cytokines and nitric oxide by human mast cells incubated with toxoplasma gondii lysate. Korean J Parasitol 57:201-206. https://doi.org/10.3347/kjp.2019.57.2.201

Pego B et al (2019) Schistosoma mansoni coinfection attenuates murine toxoplasma gondii-induced crohn's-like ileitis by preserving the epithelial barrier and downregulating the inflammatory response. Front Immunol 10:442. https://doi.org/10.3389/fimmu .2019 .00442

Peristeris P et al (1992) N-acetylcysteine and glutathione as inhibitors of tumor necrosis factor production. Cell Immunol 140:390-399. https://doi.org/10.1016/0008-8749(92)90205-4

Polari LP, Carneiro PP, Macedo M, Machado PRL, Scott P, Carvalho EM, Bacellar O (2019) Leishmania braziliensis infection enhances toll-like receptors 2 and 4 expression and triggers TNF-alpha and IL-10 production in human cutaneous leishmaniasis. Front Cell Infect Microbiol 9:120. https://doi.org/10.3389/fcimb.2019.00120

Sampaio EP, Sarno EN, Galilly R, Cohn ZA, Kaplan G (1991) Thalidomide selectively inhibits tumor necrosis factor alpha production by stimulated human monocytes. J Exp Med 173:699-703. https ://doi.org/10.1084/jem.173.3.699

Schwartz J et al (2018) Combination of paromomycin plus human antiTNF-alpha antibodies to control the local inflammatory response in BALB/ mice with cutaneous leishmaniasis lesions. J Dermatol Sci 92:78-88. https://doi.org/10.1016/j.jdermsci.2018.07.005

Thapa RJ et al (2016) DAI senses influenza A virus genomic RNA and activates RIPK3-dependent cell death. Cell Host Microbe 20:674-681. https://doi.org/10.1016/j.chom.2016.09.014 
Upton JW, Kaiser WJ, Mocarski ES (2019) DAI/ZBP1/DLM-1 complexes with RIP3 to mediate virus-induced programmed necrosis that is targeted by murine cytomegalovirus vIRA. Cell Host Microbe 26:564. https://doi.org/10.1016/j.chom.2019.09.004

Wan S et al (2018) Chi313: a potential key orchestrator of eosinophil recruitment in meningitis induced by Angiostrongylus cantonensis. J Neuroinflamm 15:31. https://doi.org/10.1186/s1297 4-018-1071-2

Wang R, Jaw JJ, Stutzman NC, Zou Z, Sun PD (2012) Natural killer cell-produced IFN-gamma and TNF-alpha induce target cell cytolysis through up-regulation of ICAM-1. J Leukoc Biol 91:299-309. https://doi.org/10.1189/jlb.0611308

Wang H et al (2016) MiR-124 regulates apoptosis and autophagy process in MPTP model of Parkinson's Disease by targeting to bim. Brain Pathol 26:167-176. https://doi.org/10.1111/bpa.12267

Wang Y, Welc SS, Wehling-Henricks M, Tidball JG (2018) Myeloid cell-derived tumor necrosis factor-alpha promotes sarcopenia and regulates muscle cell fusion with aging muscle fibers. Aging Cell 17:e12828. https://doi.org/10.1111/acel.12828

Wang Y et al (2019) Necroptosis regulates tumor repopulation after radiotherapy via RIP1/RIP3/MLKL/JNK/IL8 pathway. J Exp Clin Cancer Res 38:461. https://doi.org/10.1186/s13046-019-1423-5

Yan LZ et al (2018) The opposite roles of PAS-5 and Galectin-1 in immune response during the early infection of Angiostrongylus cantonensis. Parasit Vectors 11:318. https://doi.org/10.1186/s1307 1-018-2894-5

Ye C, Bhan AK, Deshpande V, Shankar P, Manjunath N (2013) Silencing TNF-alpha in macrophages and dendritic cells for arthritis treatment. Scand J Rheumatol 42:266-269. https://doi. org/10.3109/03009742.2013.777779

Yoshida T et al (1996) Defective B-1 cell development and impaired immunity against Angiostrongylus cantonensis in IL-5R alphadeficient mice. Immunity 4:483-494. https://doi.org/10.1016/ s1074-7613(00)80414-8
Yu X, He S (2016) The interplay between human herpes simplex virus infection and the apoptosis and necroptosis cell death pathways. Virol J 13:77. https://doi.org/10.1186/s12985-016-0528-0

Yuan J, Amin P, Ofengeim D (2019) Necroptosis and RIPK1-mediated neuroinflammation in CNS diseases. Nat Rev Neurosci 20:19-33. https://doi.org/10.1038/s41583-018-0093-1

Zamboni DS, Lima-Junior DS (2015) Inflammasomes in host response to protozoan parasites. Immunol Rev 265:156-171. https://doi. org/10.1111/imr.12291

Zelova H, Hosek J (2013) TNF-alpha signalling and inflammation: interactions between old acquaintances. Inflamm Res 62:641-651. https://doi.org/10.1007/s00011-013-0633-0

Zhang L, Feng Q, Wang T (2018) Necrostatin-1 protects against paraquat-induced cardiac contractile dysfunction via RIP1RIP3-MLKL-dependent necroptosis pathway. Cardiovasc Toxicol 18:346-355. https://doi.org/10.1007/s12012-017-9441-z

Zheng M, Wu Z, Wu A, Huang Z, He N, Xie X (2016) MiR-145 promotes TNF-alpha-induced apoptosis by facilitating the formation of RIP1-FADDcaspase-8 complex in triple-negative breast cancer. Tumour Biol 37:8599-8607. https://doi.org/10.1007/s1327 7-015-4631-4

Zhou H et al (2019) Necroptosis and caspase-2-mediated apoptosis of astrocytes and neurons, but not microglia, of rat hippocampus and parenchyma caused by Angiostrongylus cantonensis infection. Front Microbiol 10:3126. https://doi.org/10.3389/fmicb .2019 .03126

Zhou Y, Shao A, Yao Y, Tu S, Deng Y, Zhang J (2020) Dual roles of astrocytes in plasticity and reconstruction after traumatic brain injury. Cell Commun Signal 18:62. https://doi.org/10.1186/s1296 4-020-00549-2

Publisher's Note Springer Nature remains neutral with regard to jurisdictional claims in published maps and institutional affiliations. 